\title{
Aging interacts with tumor biology to produce major changes in the immune tumor microenvironment
}

Rossin Erbe ${ }^{1,2}$, Zheyu Wang ${ }^{2,3}$, Neeha Zaidi², Michael Topper ${ }^{2}$, Stephen Baylin², Elizabeth M Jaffee $^{2}$, Hariharan Easwaran ${ }^{2, *}$, Elana J Fertig ${ }^{2,4,5, *}$

\section{Affiliations}

1 McKusick-Nathans Institute of the Department of Genetic Medicine, Johns Hopkins School of Medicine, Baltimore, MD, USA

2 Department of Oncology, Sidney Kimmel Comprehensive Cancer Center, Johns Hopkins School of Medicine, Baltimore, MD, USA

3 Department of Biostatistics, Johns Hopkins Bloomberg School of Public Health, Baltimore, MD, USA

4 Department of Applied Mathematics and Statistics, Johns Hopkins University Whiting School of Engineering, Baltimore, MD, USA

5 Department of Biomedical Engineering, Johns Hopkins Bloomberg School of Medicine, Baltimore, MD, USA

* Corresponding Author/Lead Contact

Corresponding Author:

Elana J. Fertig, PhD

Assistant Professor, Oncology

The Johns Hopkins University School of Medicine

Phone: (410)-955-4268

ejfertig@jhmi.edu 


\section{Abstract}

2 Advanced age is strongly correlated with both increased cancer incidence and general immune

3 decline. The immune tumor microenvironment (ITME) has been established as an important

4 prognostic of both therapeutic efficacy and overall patient survival. Thus, age-related immune

5 decline is an important consideration for the treatment of a large subset of cancer patients.

6 Current studies of aging-related immune alterations are predominantly performed on non-

7 cancerous tissue, requiring additional study into the effects of age on tumor immune infiltration.

8 We leverage large scale transcriptional data sets from The Cancer Genome Atlas and the

9 Genotype-Tissue Expression project to distinguish normal age-related immune alterations from

10 age-related changes in tumor immune infiltration. We demonstrate that while there is overlap

11 between the normal immune aging phenotype and that of the ITME, there are several changes

12 in immune cell abundance that are specific to the ITME, particularly in T cell, NK cell, and

13 Macrophage populations. These results suggest that aged immune cells are more susceptible to

14 tumor suppression of cytotoxic immune cell infiltration and activity than normal tissues, which

15 creates an unfavorable ITME in older patients in excess of normal immune decline with age and

16 may inform the application of existing and emerging immunotherapies for this large population

17 of patients. We additionally identify that age-related increases in tumor mutational burden are

18 associated with decreased DNA methylation and increased expression of the immune

19 checkpoint genes PDL1, CD80, and LAG3 which may have implications for therapeutic

20 application of immune checkpoint blockade in older patients.

\section{Introduction}

23 The association of cancer incidence with age is well established and the phenomenon of age-

24 related immune decline has been recognized for even longer (Gardner, 1980). Mutations and

25 epigenetic alterations are believed to accumulate with age and drive carcinogenesis (Tomasetti

26 et al., 2017), (Horvath, 2013). Recent research has highlighted the specific changes that 
1 contribute to the general decline of the immune system that occurs as individuals age (Aw et al.,

2 2007). Understanding the effect such alterations have on the anti-tumor immune response is

3 critical for the informed development and application of immunotherapies to elderly patients.

5 Thus, systemic immune aging has received considerable attention in the context of its effect on

6 cancer development and progression (Fulop et al., 2017). In particular, loss of T cell receptor

7 (TCR) diversity (Britanova et al., 2014), decreased capacity of cytotoxic cells (Solana and

8 Mariani, 2000), and increased inflammatory signaling (Franceschi et al., 2000) have been

9 identified as age-related immune changes of potential relevance to cancer therapeutics and

10 patient survival. Currently, these age-related changes in the immune system are identified

11 predominantly in non-cancerous tissues. However, tumors actively alter immune cells and their

12 immune microenvironment to promote disease progression and avoid targeting by the immune

13 system, which has been identified as one of the major hallmarks of cancer (Hanahan and

14 Weinberg, 2011). Therefore, additional aging-related shifts may occur in the immune tumor

15 microenvironment (ITME) as a result of interactions between tumor immunosuppressive

16 signaling and the immunosenescence phenotype.

18 In the last decade, the composition of the ITME has become a subject of intense study due to its 19 association with overall survival and therapeutic efficacy, particularly that of immune checkpoint 20 blockade (ICB) (Frankel et al., 2017). However, any shifts in the composition of the ITME itself

21 that may occur with age have not yet been generally characterized, with the exception of some

22 T cell populations in melanoma (Kugel et al., 2018). Merging the disparate research on cancer

23 and aging can further distinguish whether age-related changes in non-cancerous tissues are

24 recapitulated within the ITME. At present, ICB immunotherapy is less often used to treat elderly

25 patients, due to concerns about efficacy and toxicity, despite the fact that the limited clinical trial

26 data that exists suggests that they experience no reduced benefit as compared to younger 
1 patients (Kugel et al., 2018), (Elias et al., 2018), (Daste et al., 2017), (Jain et al., 2019). Age-

2 specific characterization of the ITME is essential to understand these results and move forward

3 with efforts to bring immunotherapy to this large group of cancer patients.

5 This study directly compares the impact of aging on immune response and infiltration in tumors

6 to that of non-cancerous tissues using genomics data from large-scale consortium studies. We

7 apply immune cell type deconvolution methods to bulk transcriptomics data from untreated

8 tumors samples among patients of different ages from The Cancer Genome Atlas (TCGA) and

9 to post-mortem non-cancer tissue samples from individuals of different ages from the Genotype-

10 Tissue Expression project (GTEx) in order to compare age-associated immune composition

11 changes from within tumors and non-cancerous tissues. We identify decreases in overall $\mathrm{T}$ cell

12 abundance in tumor samples that are not observed in systemic tissues, as well as an increase

13 in macrophage abundance. Further, while NK abundance generally increases with age, this

14 trend is not observed within the ITME. These cancer-specific changes are both poor prognostics

15 based on TCGA overall survival data and the existing literature on the impact of these immune

16 cell types in the ITME. These analyses suggest that not only do older cancer patients face the

17 normal aspects of immune decline, but that there is a specific interaction between the senescent

18 immune system and tumor signaling that produces a less favorable ITME for survival and

19 therapeutic response. This represents an unappreciated interaction between tumor biology and

20 the aging immune system that contributes to the worsening of clinical outcomes with increasing

21 age and may suggest new treatment strategies for elderly patients. At the same time, we

22 observe that increasing tumor mutational burden with age appears to lead to an epigenetically

23 regulated increase in tumor expression of immune checkpoint receptors such as $P D L 1, C D 80$,

24 and $L A G 3$, which may make ICB an unexpectedly attractive therapeutic option for many older

25 patients. 


\section{Results}

2 Deconvolution of immune cell abundance in tumor samples from TCGA reveals an age-related

3 decrease in T cell abundance and increase in macrophage abundance that is associated with

\section{4 decreased survival}

5 The large number of primary tumor transcriptional profiles across disease subtypes available

6 from TCGA provides a unique cohort to characterize the impact of age on the ITME. We apply

7 the MIXTURE immune cell type deconvolution algorithm (Fernandez et al., 2019) to infer the

8 absolute proportions of immune cell types from RNA-sequencing data derived from pan-cancer

9 TCGA samples. We then fit a linear model with age, including cancer type (based on the 33

10 TCGA cancer-type projects included in the study) and patient sex as covariates, for each

11 immune cell type (listed in Supplemental Table 1) to assess changes in immune cell infiltration

12 as patients age. We find that overall $\mathrm{T}$ cell abundance significantly decreases with age in the

13 ITME (q-value $=0.00175)($ Figure $1 \mathrm{~A})$ while macrophages significantly increase in abundance $(\mathrm{q}$

$\left.14=4.45 \times 10^{-4}\right)($ Figure $1 \mathrm{~B})$. Detectable changes in the infiltration of NK cells, Dendritic cells, B

15 cells, and other myeloid populations do not occur with age pan-cancer (Figure 1C, Table 1).

17 We additionally investigate age-related changes in the TCGA cohort at a finer cellular resolution

18 of 22 immune cell subtypes from MIXTURE (listed in Supplemental Table 1) (Supplemental

19 Figure 1). Naive B cells are found to significantly decrease in abundance with increasing age in

20 the ITME (q-value $=0.0305)$. Although many known systemic immune changes occur with age

21 in healthy tissues, this reduction in Naive B cells is the only statistically significant shift among

22 immune cell subtypes in the ITME. Consistent with our previous analysis of the higher order cell

23 types, the three macrophage subsets (M0, M1, and M2) are among the top four cell types that

24 trend towards increasing abundance in age. Likewise, all T cell subsets (CD8, CD4, Follicular

25 helper, and regulatory T cells) trend towards a decrease in abundance with age with the

26 exception of gamma delta T cells. 
2 To investigate the association of these age-related immune infiltration changes with patient

3 survival, we fit a Cox proportional hazards model between overall survival and all immune cell

4 types (both subtypes and higher order cell types), adjusting for patient age, sex, cancer type,

5 and years of smoking (Supplemental Figure 2, Table 2). We observe that higher risk is

6 significantly associated with lower T cell abundance $\left(q=5.38 \times 10^{-4}\right)$ and NK cell abundance ( $q$

$7=0.0263)$, as well as for higher macrophage abundance $(q=0.00162)$. Naive $B$ cells were not

8 significantly associated with survival $(q=1.00)$.

10 We note that both the composition of the ITME and average patient age varies by cancer type.

11 Therefore, to determine the variance in age-related effects that occur within different cancer

12 types, we then evaluate the association between age and immune composition for each cancer

13 type with at least 100 samples that could be successfully deconvolved by the MIXTURE

14 algorithm (Supplemental Figure 3). We identify considerable heterogeneity in the effect of age

15 on immune cell type abundance across cancer types, though macrophages generally increase

16 in abundance, while T cells generally decrease (Figure 2A), consistent with the results of our

17 pan-cancer analysis. Breast carcinomas have the most significant decrease in $\mathrm{T}$ cell abundance

18 and the most significant increase in macrophage abundance with age overall.

20 To further distinguish the relative role of age and cellular composition of the ITME with patient

21 outcomes, we then fit a Cox proportional hazards model for the effects of variation in each

22 immune cell type across cancer types, including diagnosis age as a covariate (Figure 2B).

23 Macrophages and other myeloid-derived cells are generally poor prognostics across cancer

24 types, while $\mathrm{T}$ and NK cells are generally good prognostics. Head and neck squamous cell

25 carcinomas (HNSC) have the most significant survival association with both macrophages and

26 T cells. If HNSC cases are subdivided into HPV-negative and HPV-positive patients, this 
1 association only recapitulates among the HPV-negative cohort, emphasizing the importance of

2 ITME composition for HPV-negative HNSC cases.

$4 \quad$ Age-related immune changes in non-cancerous tissues differ from the observed shifts in the

5 aging ITME among T cells, macrophages, B cells, and NK cells

6 To compare the effect of aging in the ITME to that on the immune cell compositions of normal

7 tissues, we applied MIXTURE to GTEx consortium RNA-sequencing data of post-mortem

8 samples from individuals without cancer (GTEx Consortium et al., 2017) to infer cell type

9 abundance across tissues. These results provide a non-cancer baseline for immune changes

10 that occur across many individuals of varying ages to compare with our observations from

11 TCGA tumor data. Similar to the TCGA analysis, we fit a linear model to each cell type in order

12 to determine associations between cell type abundance and age both across and within normal

13 tissues.

15 In contrast to our findings in the pan-cancer ITME, in pan-tissue analyses we observe no 16 significant change in overall T cell abundance with age $(q=0.565)$ (Figure 3A, Supplemental

17 Table 2). We further fail to find significant changes in macrophage levels $(q=0.565)$ with age

18 (Figure 3B). However, we do observe decreases in overall B cell $\left(q=3.51 \times 10^{-4}\right)$ (Figure 3C)

19 proportion and increases in NK cell proportion $\left(q=1.07 \times 10^{-14}\right)$ (Figure 3D). This result

20 demonstrates that there are considerable differences between the effects of aging on the

21 abundance of immune cells in non-cancer tissues and the ITME.

23 Across the 22 immune cell subtypes from MIXTURE, we find that naive $B$ cell abundance is

24 significantly decreased in GTEx samples with increasing age, consistent with our findings in the

25 ITME (q-value of $9.07 \times 10^{-16}$ and 0.0305 , respectively). However, among GTEx data, we also

26 observe significant decreases in memory CD4 T cells $\left(q=3.30 \times 10^{-7}\right)$, naive CD4 T cells $(q=$ 
$0.00604)$, M2 macrophages $\left(q=3.59 \times 10^{-5}\right)$, memory B cells $(q=0.0315)$, and resting dendritic

2 cells $(q=0.0427)$, as well as significant increases in abundance of resting NK cells $(q=5.33 x$

$\left.310^{-11}\right)$ and CD8 T cells $(q=0.0157)$ (Figure 3E). These findings represent clear departures from

4 what we observe within the ITME, suggesting that tumors interact with the aging immune

5 system to selectively prevent or increase infiltration of certain immune cell types that exist

6 systemically, in a way that differs from the tumor immunosuppression that occurs in younger

7 patients.

8

9 To directly compare normal age-related immune changes in a particular tissue to those that

10 occur in tumors within the same tissue, we individually assay the immune associations with age

11 for colon, lung, and breast tumors in TCGA with those found for all normal colon, lung, and

12 breast samples from GTEx (Figure 3F, Supplemental Figure 4). Consistent with what we find

13 pan-cancer and pan-tissues, we observe an increase in macrophage infiltration and a decrease

14 in T cell infiltration among colorectal adenocarcinoma patients, while there is no significant

15 change observed for colon samples from GTEx, and the non-significant trend that we do

16 observe is reversed for both cell types. Similar immune cell type differences are found between

17 cancerous and non-cancerous tissues in lung and breast samples. These results demonstrate

18 that systemic age-related immune changes cannot be assumed to translate to the ITME.

19 Particularly, they suggest that an interaction occurs between the phenotype of aging immune

20 cells and the immunosuppressive signaling of cancers that generally increases the infiltration of

21 macrophages and decreases the infiltration of T cells and NK cells in older patients.

23 Systemic age-related increases in M1/M2 macrophage ratio and decreases in CD8/CD4 T cell

24 ratio in non-cancerous tissues do not recapitulate in the ITME

25 We hypothesize that the tumor-specific changes to the ITME with age are associated with the 26 modifications that cause tumors to evade immune attack. A higher ratio of M1/M2 macrophages 
1 has been previously found to be a positive survival prognostic (Chanmee et al., 2014), which we

2 evaluate using a Cox proportional hazards model for overall survival in TCGA. We find that an

3 increased M1/M2 ratio is a generally good prognostic pan-cancer, including age and cancer

4 type as covariates $(q=0.0165)$. As the trends displayed in Figure 3E would suggest, the M1/M2

5 macrophage ratio significantly increases $\left(q=4.12 \times 10^{-4}\right)$ with age within normal tissues from

6 GTEx data (Supplemental Figure 5A). By contrast, the M1/M2 tumor infiltration ratio does not

7 change with age pan-cancer in tumor tissues in TCGA data $(q=0.314)$ (Supplemental Figure

$85 \mathrm{~B})$, consistent with a signature of decreased immune activation relative to the rest of the body.

9 Likewise, greater T cell killing would be expected to be associated with better prognosis. In

10 TCGA, CD8/CD4 ratio has a minor favorable association with patient survival which falls short of

110.05 statistical significance $(q=0.0977)$. We observe an increase in the CD8/CD4 T cell ratio

12 with age in GTEx samples $\left(q=2.87 \times 10^{-15}\right)$ (Supplemental Figure $\left.5 \mathrm{C}\right)$ that does not recapitulate

13 within the ITME ( $q=0.314)$ (Supplemental Figure 5D).

T cell receptor clonality decreases with age within the ITME while the number of tumor

16 mutations increases

17 The overall decline in the total number of unique T cell receptor (TCR) clones with age (Yager et al., 2008), (Britanova et al., 2014), (Egorov et al., 2018) is well established in the literature. The process of thymic involution (the loss of thymus tissue with age) eventually ends the production

20 of naive T cells and is the major driver of normal age-related decreases in $\mathrm{T}$ cell clonality

21 (Aspinall and Andrew, 2000). To quantify aging-related changes in TCR clonality in the ITME, 22 we leveraged results previously generated with the miTCR algorithm (Bolotin et al., 2013) by

23 (Thorsson et al., 2018) to determine the association between TCR clonality and age. We define

24 our metric of clonal diversity as the Shannon entropy multiplied by the number of unique clones

25 divided by the total number of TCR sequencing reads (this correction is important because we 26 have already established that T cell abundance decreases with age and we wish to correct this 
1 metric for the expected lower number of T cells to be sequenced among the samples from older

2 patients). We determine that this TCR clonality measure significantly decreases with age for

3 pan-cancer TCGA samples, including cancer type as a covariate $\left(p=1.48 \times 10^{-8}\right)($ Table 3 ,

4 Supplemental Figure 6A). We then fit a Cox proportional hazards model with age and cancer

5 type as covariates and determine that decreased TCR clonality is a significant negative

6 prognostic for overall survival $\left(p=3.34 \times 10^{-5}\right)($ Table 3$)$. This result suggests that the reduced

7 ability to recognize antigens in older individuals leads to reduced T cell killing of tumor cells and

8 hence worse outcomes.

10 However, a related consideration is the increasing accumulation of mutations known to occur

11 with age (Tomasetti et al., 2017) and the potential accompanying increase in immunogenic

12 mutations. Higher tumor mutational burden has been shown to correlate with improved

13 outcomes in ICB immunotherapy (Yarchoan et al., 2017), (Goodman et al., 2017), which

14 suggests the possibility that the increasing number of mutations accumulated with age may in

15 some cancers offset the loss of TCR diversity. As has been previously reported (Chalmers et

16 al., 2017), (Qing et al., 2020), we find that tumor mutational burden significantly increases with

17 age $\left(p<1 \times 10^{-16}\right)$ (Supplemental Figure 6B) pan-cancer in TCGA, including cancer type as a

18 covariate (Table 3). This trend recapitulates within most cancer types, though lung

19 adenocarcinomas and uterine carcinomas are notable exceptions (Supplemental Figure 6C).

20 High mutational burden among younger patients with lung cancer is likely due to the highly

21 mutagenic effects of cigarette smoking, while the highly mutated uterine tumors are likely the

22 result of a hypermutated subset previously discovered among the TCGA-UCEC cohort (Cancer

23 Genome Atlas Research Network et al., 2013). A higher tumor mutational burden is a positive

24 survival prognostic pan-TCGA, as determined by a Cox proportional hazards model, including

25 age and cancer type as covariates $(p=0.011)$ (Table 3$)$. Thus, while the ability to recognize

26 antigens may decrease with age, the space of tumor antigens for a given TCR to match with will 
1 likely increase, presumably at least partially offsetting the decreased T cell capacity to recognize

2 tumors caused by loss of TCR diversity.

4 Age-related increases in tumor mutational burden associates with promoter demethylation and

$5 \quad$ increased expression of immune checkpoint genes in tumor samples

6 The ability of TCR sequences to recognize tumor antigens is of particular clinical relevance in

7 the context of ICB immunotherapy. Another important factor for the efficacy of ICB

8 immunotherapy is the expression of target genes and their complementary receptors such as

9 PD1, PDL1, CTLA4, CD80, and CD86 (Taube, 2014). We therefore performed differential

10 expression analysis for these genes with age in both TCGA tumor samples and normal GTEx

11 tissue samples, including cancer type and tissue type as respective covariates. Among TCGA

12 samples, $C D 80$ and $P D L 1$ expression increases with age (q-values 0.0116 and 0.0299 ), while

13 no detectable expression changes occur with age for CTLA4, PD1, and CD86 (q-values of

$140.779,0.693$, and 0.0834 ) (Table 4), suggesting that older patients display increased tumor cell

15 expression of these immune checkpoint genes. Another possibility is that this change occurs

16 due to the increased number of infiltrating macrophages we have shown accumulate with age,

17 as PDL1 and CD80 can be expressed on macrophages as well (Hartley et al., 2018). We further

18 investigate age-related changes in expression of these genes within each cancer type

19 (Supplemental Figure 7). There is considerable heterogeneity in the effect of age on the

20 expression of these genes, though $P D L 1, C D 80$, and $C D 86$ are more likely to increase in

21 expression with age, while PD1 and CTLA4 expression is more variable, suggesting that tumor

22 expression of immune checkpoint genes is more affected by age than tumor infiltrating $\mathrm{T}$ cell

23 expression of immune checkpoint genes.

25 Among the non-cancerous samples from GTEx, PD1 and PDL1 expression significantly

26 increases with age (q-values of $2.93 \times 10^{-7}$ and 0.00166$), C D 86$ expression does not 
1 significantly change $(q=0.891)$, and there was no data available for CTLA4 or CD80

2 (Supplemental Data). The increase in PD1 expression is possibly due to the increased presence

3 of exhausted CD8 T cells, which have been previously reported to accumulate with age and

4 express both PD1 and TIM3 (Lee et al., 2016), the latter of which is also expressed at increased

5 levels with advanced age in GTEx data $\left(q=2.157 \times 10^{-5}\right)$. The increased inflammation observed

6 in older individuals (Fulop et al., 2017), (Kovtonyuk et al., 2016) may explain the increased

7 expression of $P D L 1$ in older individuals from GTEx. Among the inflammatory pathways up with

8 age, we particularly note that the GO term for response to interferon gamma significantly

9 increases in GTEx ( $q=0.00297)$, which has been reported to stimulate PDL1 expression (Flies

10 and Chen, 2007).

12 Tumor mutational burden is an important clinical predictor of response to immunotherapy that

13 increases with patient age. To determine if these age-related changes in immune checkpoint

14 gene expression are associated with the increase in tumor mutational burden that we described

15 previously (Supplementary Figure 6B), we included it as a covariate and repeated the

16 differential expression analysis. PDL1 expression no longer appeared significantly associated

17 with age $(q=0.207)$, while $C D 80$ expression became borderline $(q=0.0502)$ (Supplemental

18 Data). These data indicate that increased expression of $P D L 1$ with age most directly associates

19 with age-related increases in tumor mutational burden.

21 Due to previous work suggesting that DNA methylation regulates tumor expression of PDL1

22 (Asgarova et al., 2018), (Micevic et al., 2019), we hypothesize that the observed expression

23 increases of immune checkpoint genes are largely driven by epigenetic changes. We leverage

24 merged 450k and 27k methylation array data from TCGA (Thorsson et al., 2018) and find that

25 methylation of CpGs annotated to the PDL1 promoter region significantly decreases with age

26 pan-cancer $\left(q=3.27 \times 10^{-10}\right)$, including cancer type as a covariate. Methylation of CTLA4 and 
$1 C D 80$ annotated CpGs also decrease with age (q values of $4.33 \times 10^{-4}$ and $6.01 \times 10^{-5}$ ) (Table

2 4). These results suggest that DNA methylation changes lead to the observed expression

3 increases in PDL1 and CD80 with increasing age and that age-related increases in tumor

4 mutational burden promote selective pressure for epigenetically mediated up-regulation of

$5 \quad P D L 1$.

6

$7 \quad$ Corresponding shifts in DNA methylation and expression in TCGA samples suggest profound

8 changes in the aging tumor microenvironment

9 In order to further investigate the role of age-related methylation changes on the ITME, we

10 identified all genes annotated to have some immune role by InnateDB (Breuer et al., 2013)

11 within TCGA expression and methylation data. In order to find immune genes that are regulated

12 by age-related methylation changes at promoters of these genes, we then selected the following

13 two sets of differentially expressed genes: those that had significant increases in expression

14 and significant decreases in methylation with age, and those with significant decreases in

15 expression and significant increases in methylation with age. We note that increased

16 methylation of annotated promoter CpGs was much more likely to indicate that the

17 corresponding gene would decrease in expression (218 anti-correlated vs 66 correlated with

18 age) than decreased methylation was to indicate that a gene's expression would increase (113

19 correlated vs 94 anti-correlated with age) (Supplemental Table 3).

21 Among the genes with increased expression and decreased methylation in TCGA, we find the

22 GO immune regulation term $\left(q=5.90 \times 10^{-25}\right)$, the regulation of $T$ cell activation term $(q=2.93 \times$

$\left.2310^{-5}\right)$, and innate immune response term $\left(q=1.74 \times 10^{-11}\right)$ significantly enriched (Figure 4A) (the

24 full list of GO enrichments is available in Supplemental Data). Of particular note is the

25 connected group of T cell regulatory genes PDL1, CD80, LAG3, HAVRC2, and IL10. LAG3 has

26 been shown to be of importance in immune infiltration and overall survival in renal cell 
carcinoma (Klümper et al., 2020), acts as an important player in intratumoral T cell exhaustion in

2 lymphoma (Yang et al., 2017), and has been suggested as a potential therapeutic target for new

3 ICB strategies (Long et al., 2018). HAVRC2 (also known as TIM3) has been shown to be an

4 important inhibitory $\mathrm{T}$ cell receptor, as well as a defining characteristic of exhausted T cells in

5 concert with PD1 (Lee et al., 2016), (Wolf et al., 2020). IL10 has been shown to be a direct

6 inhibitor of CD8 T cell function (Smith et al., 2018). We thus observe a set of methylation-

7 associated expression changes with increasing age that are expected to have a considerable

8 detrimental effect on T cell tumor killing.

10 Next, we investigated if these methylation and expression changes are related to the age-

11 associated TMB changes, like we observed for $P D L 1$ and $C D 80$. We performed the same

12 analysis of immune-related genes in InnateDB, adjusting for tumor mutational burden for both

13 differential expression and methylation, and only IL 10 retains significantly increased expression

14 and decreased methylation of the aforementioned five genes (Figure 4B). Therefore, the effect

15 of age on T cell activation in our model seems to be largely explained by age-related changes in

16 tumor mutational burden, while other age-related factors have a larger impact on the increased

17 expression of a number of genes related predominantly to innate immunity. For example,

18 another 9 genes annotated to the innate immune response GO term are significant for the TMB

19 corrected analysis (25 vs 34 total).

21 The genes with increased CpG methylation and decreased expression with age in TCGA

22 samples are significantly enriched for a number of GO terms, notably including cell adhesion (q

$\left.23=9.38 \times 10^{-44}\right)$, cell differentiation $\left(q=5.83 \times 10^{-31}\right)$, locomotion $\left(q=1.84 \times 10^{-21}\right)$, and

24 extracellular matrix organization $\left(q=6.18 \times 10^{-21}\right)$ (Figure $\left.4 \mathrm{C}\right)$ (full list provided in Supplemental

25 Data). This gene set also includes several growth factors and receptors with major known roles

26 in tumor biology, such as EGFR (Normanno et al., 2006), FGF2 (Soufla et al., 2005), PDGFRA 
1 (Velghe et al., 2014), and IGF1R (Pandini et al., 1999). All of these terms and genes remain

2 significant after correcting for tumor mutational burden (Figure 4D), though major transcription

3 factors such as SOX4 and FOXO1 drop out, as do the growth factors TGFB2 and NGF,

4 indicating that high mutational burden may play a role in age-related gene methylation for a

5 subset of these genes. However, most of these gene expression and methylation changes

6 appear to be mediated through other age-related effects. Epigenetic silencing of these pathways

7 and genes would be expected to have a considerable effect on the tumor microenvironment, as

8 they play major roles in critical tumor processes such as growth, metastasis, dedifferentiation,

9 and angiogenesis.

11 GO gene set enrichment differences between TCGA and GTEx data with age suggest

12 interactions of tumor signaling and aging biology produce substantial changes in tumor cellular

13 processes and microenvironment

14 To further investigate possible causes for the observed differences in immune infiltration

15 between aged tumors and healthy aged tissues, we compare GO term enrichment based on

16 gene expression changes with age from TCGA and GTEx samples. We primarily investigate

17 four sets of GO terms: those up in both TCGA and GTEx with age, those up in TCGA and down

18 in GTEx, those up in GTEx and down in TCGA, and those down in both TCGA and GTEx

19 (Supplemental Figure 8) (full list of GO terms provided in Supplemental Data). The pathways up

20 in both are highly immune related, indicating an increase in inflammation with age that has

21 previously been termed inflammaging (Fulop et al., 2017), (Franceschi et al., 2000). The

22 increases in antigen binding, MHC complex, and Interferon gamma signaling terms are of

23 relevance to $\mathrm{T}$ cell recognition and activity. Of particular note may be the increase in Interferon

24 gamma signaling, which is known have a role upregulating PDL1 expression (Flies and Chen,

25 2007), and thus may be involved in the expression increase of $P D L 1$ with age, along with the

26 previously noted relation to tumor mutational burden. Among those pathways up in TCGA and 
1 down in GTEx, the Mitochondrion term seems the most potentially relevant, as energetics is a

2 major factor in tumor biology, and mitochondria additionally play a considerable role in

3 regulating cell death pathways as well as immune regulation (Weinberg et al., 2015), (Breda et

4 al., 2019). Regulation of proliferation, positive regulation of development, locomotion, and

5 biological adhesion are up in GTEx and down in TCGA, with potential relevance for tumor

6 growth and metastasis. These terms match closely with the terms found to be silenced by DNA

7 methylation with age in the previous section, indicating that these are cancer specific age-

8 related effects as well. There thus appears to be another interaction of aging biology and tumor

9 signaling that may alter the development of several major hallmarks of cancer in older patients.

10 Finally, cell cycle terms are down in both cohorts with increasing age. In the context of the

11 altered age-related immune infiltration in tumors compared to normals, these data demonstrate

12 that there are inflammation increases with age in both tumor and healthy aged tissues. Thus,

13 the observed changes in immune cell abundance between the tumors of old patients and the

14 tissues of old individuals are likely the result of an interaction between tumor

15 immunosuppressive signaling and the altered phenotype of aged immune cells.

\section{Discussion}

18 We demonstrate that patient age associates with several changes in ITME composition that do

19 not occur in non-cancerous tissues. This result indicates that tumor signaling interacts with the

20 aging immune system and/or environment to modulate immune infiltration and produce a more

21 immunosuppressive microenvironment in older patients than would be predicted by normal

22 immune aging in isolation. We further investigate the complex interplay of age-related shifts in

23 TCR clonality, tumor mutational burden, and T cell exhaustion that appear to modulate the

24 demethylation and increased expression of immune checkpoint genes in the tumors of older

25 patients. 
1 In contrast to our analysis of ITME cell type composition shifts with age, there is an existing

2 literature on normal age-related immune changes, which allows for validation of the results of

3 the MIXTURE method in this work with an established base of knowledge. The observed

4 decreases in overall $\mathrm{T}$ cell abundance within tumors did not recapitulate in normals from GTEx

5 and have not been reported to occur systemically. While this may seem a strange phenomenon

6 given the process of thymic involution, it has been previously found that it is mostly naive T cells

7 that decrease in abundance with age, while the population of memory T cells proportionally

8 increases (with accumulation of exhausted, less functional effector T cells as individuals reach

9 very advanced age) (Hulstaert et al., 1994), (Alpert et al., 2019). Thus, overall T cell abundance

10 remains relatively constant, as we observe in our analysis of GTEx data. This ITME specific

11 departure may have implications for T cell based immunotherapies in elderly patients,

12 suggesting the possibility of targeting the as of yet unknown mechanism repressing infiltration to

13 potentially improve therapeutic efficacy in older patients.

15 We observe increased infiltration of macrophages in tumor samples, without a particular bias in

$16 \mathrm{M} 1 / \mathrm{M} 2$ polarization, while in normal GTEx data we identify no change in macrophage

17 abundance overall, but find that the M1/M2 ratio increases. Previous studies have suggested

18 that old age alters macrophage polarization such that the same signals that would produce

19 polarization in younger individuals do not produce differentiated macrophages with all the

20 characteristics generally ascribed to M1 and M2 subtypes (Mahbub et al., 2012). Thus, these

21 results must be interpreted carefully, but an increase in tumor infiltrating macrophages still

22 appears to be a poor survival prognostic in older individuals, which suggests that therapeutics

23 being developed to target the generally negative effects of tumor-associated macrophages

24 (Chanmee et al., 2014), (Poh and Ernst, 2018), (Lee et al., 2019) may be of particular impact in

25 patients of advanced age. 
1 A further observation of note is that we find a significant increase in NK cell abundance in GTEx,

2 which we do not identify in TCGA tumor data. It has been previously shown that overall NK cell

3 abundance increases with age, though their cytotoxicity was diminished relative to younger

4 controls (Gounder et al., 2018). It therefore seems that NK cell tumor infiltration is inhibited with

5 increasing patient age because this general increase in abundance is not reflected in the ITME.

6 The role of NK cells in cancer immunity is gaining increasing appreciation (Freeman et al.,

7 2019), (Chiossone et al., 2018), with some evidence to suggest an important role in emerging

8 immunotherapies as well (Shimasaki et al., 2020).

10 Systemic loss of TCR clonality with age (Britanova et al., 2014) is reflected in the ITME, which,

11 along with the decreased T cell infiltration we identify, raises questions about the efficacy of

12 immunotherapies in patients of advanced age due to the value of TCR diversity as a biomarker

13 to identify patients who will benefit from ICB immunotherapy (McNeel, 2016). The possibility of

14 reduced efficacy has been partially addressed by previous studies. There are somewhat mixed

15 results as to the benefit of ICB for patients of advanced age, though most studies and meta-

16 analyses of available clinical trial data suggest patients experience no reduced benefit (Kugel et

17 al., 2018), (Elias et al., 2018), (Daste et al., 2017), (Jain et al., 2019). The observed increases in

18 tumor mutational burden with age may explain these results at least partially, as these additional

19 mutations provide a larger space of antigens for the reduced number of unique TCRs to

20 recognize. We further find that the tumor mutational burden increases with age appear to

21 mediate a decrease in methylation and increase in expression of PDL1, though we cannot rule

22 out that age-related increases in Interferon gamma signaling play a role as well. From these

23 results, we hypothesize the causal model outlined in Supplemental Figure 10, whereby TCR

24 Diversity, Tumor mutational burden, and T cell infiltration changes with age together mediate the

25 cytotoxic capacity of $T$ cells on tumors and thus the selective pressure for the expression of

26 immune checkpoint genes. Given the results of the aforementioned meta-analyses, this model 
1 suggests that increased tumor mutational burden (and the corresponding increase in PDL1

2 expression) is largely able to compensate for the loss of T cell infiltration and TCR diversity such

3 that older patients end up doing roughly as well on ICB immunotherapy as their younger

4 counterparts despite several characteristics that are generally disadvantageous, though

5 unaccounted factors may be at play as well. Currently, ICB is still used less often among elderly

6 patients (Jain et al., 2019), (Hurez et al., 2018) due to concerns about efficacy and safety. Our

7 results indicate that older individuals express increased levels of PDL1, which likely allows for

8 the equal level of response to that of younger patients. This suggests that ICB use for the

9 elderly should be further investigated and likely expanded. Additionally, our model suggests that

10 if T cell infiltration into the tumors of older patients can be increased, they might respond even

11 better to ICB immunotherapy than young patients do.

13 We identify a set of genes that are hypermethylated and lower-expressed with increasing age.

14 These genes are significantly associated with growth, metastasis, and angiogenesis, which are

15 considered to be some of the hallmarks of cancer (Hanahan and Weinberg, 2011). We further

16 find that these terms are not enriched among GTEx samples with age, demonstrating another

17 interaction between aging biology and tumor biology that produces an age-related tumor

18 phenotype that is distinct from the age-related phenotype in normal tissues. Within this set of

19 genes we observe what appears to be DNA methylation-mediated silencing of genes associated

20 with cell differentiation, which previous studies (Widschwendter et al., 2007), (Easwaran et al.,

21 2012), (Ohm and Baylin, 2007), (Schlesinger et al., 2007) indicate represents a tumor-specific

22 hypermethylation of genes that locks the constituent cells into a malignant stem-like state.

23 These changes appear to be more common in the tumors of elderly patients, suggesting

24 epigenetic therapies may be of particular value in this population. 
1 Given the age-related differences in immune infiltration observed, we identified both the

2 differences and similarities in GO term enrichment with age for TCGA and GTEx samples.

3 Inflammation and immune terms are up in both, including interferon gamma signaling, which

4 likely has some impact on the observed increase in PDL1 expression (Flies and Chen, 2007).

5 The differences in enrichment, however, are largely not directly immune related and do not

6 ostensibly explain the observed differences in immune infiltration. However, some of these

7 pathway differences, such as increased mitochondrial activity in tumors with age and decreased

8 activity in normal aged tissues, may merit future investigation due to the potential role these

9 changes may play on cellular energetics and survival.

11 To determine if age-related tumor mutational burden increases mediate the immune infiltration

12 changes we observe with age, we included it as a covariate in analyses of immune infiltration

13 derived from MIXTURE. We determined none of our observations changed substantially

14 (Supplemental Data), demonstrating that the observed differences are mediated through other

15 age-related factors. Thus, we conclude the T cell infiltration decreases, macrophage infiltration

16 increases, and NK infiltration stability despite an increasing abundance in the body overall is

17 most likely attributable to the hypothesis that exhausted or senescent phenotypes develop in

18 immune cells as individuals age and make the immune cells of older patients more sensitive to

19 the immunosuppressive signaling that is produced by most tumors (Lu et al., 2019). Another

20 possibility is that some other aspect of aging biology extrinsic to immune cells interacts with the

21 dysregulated signaling from tumors to prevent immune infiltration of certain cell types. However,

22 these investigations do not provide any particular evidence of what that actor might be and

23 therefore the former hypothesis should be favored by virtue of parsimony. Additional

24 investigation is needed to determine whether either of these proposed mechanisms drives our

25 observations, but they suggest the possibility of therapeutics targeting the immune senescence 
1 phenotype or the interaction of said phenotype and tumor immunosuppressive signaling to

2 improve outcomes for older cancer patients.

4 It is important to note the limitations of bulk expression data for the analysis of tumor-infiltrating

5 immune cells. Possibly most importantly is the consideration of immune cell function and quality

6 (e.g. the question of whether T cells are exhausted or non-functioning is difficult to address from

7 bulk data). Further, immune cell type deconvolution of bulk data does not lend itself to as

8 thorough an exploration of immune subtypes as at single-cell resolution. Therefore, future

9 single-cell pan-cancer characterization from projects such as the Human Tumor Atlas Network

10 (Rozenblatt-Rosen et al., 2020) and normal tissue through the Human Cell Atlas are critical to

11 further delineate the role that aging-related changes to immune cell function play in cancer.

12 Nonetheless, characterization of the impact aging has on the ITME from bulk data can be a

13 significant aid in the informed treatment of elderly patients.

15 Overall, these results suggest that patients of advanced age may benefit from immune

16 modulation that promotes infiltration of immune cells that are already present in their body to

17 produce a more favorable environment for therapeutic response and survival. Additionally, our

18 results, along with previous meta-analyses of clinical trials, suggest that ICB use in elderly

19 patients merits further study and expansion. Finally, these findings indicate that to fully

20 appreciate the tumor biology and treatment needs of older patients, specific study into the

21 effects of age in cancer is necessary, as we cannot necessarily rely on studies of aging in

22 general to accurately describe the effects of age in tumors.

\section{Methods}

\section{RNA-Sequencing Data}


TCGA RNA-sequencing data processed and normalized according to

2 https://docs.gdc.cancer.gov/Data/Bioinformatics Pipelines/Expression mRNA Pipeline/ was

3 downloaded from the GDC Data Portal on August 8th, 2019, filtering for all TCGA samples with

4 patients above 30 years of age. Patients under 30 were excluded to focus on ITME changes in

5 adult populations, which are more likely to generalize to the majority of cancer patients.

6

7 GTEx RNA-sequencing counts version 8 were downloaded from the GTEx Portal on November

8 12th, 2019. Only individuals over 30 were included in the final analysis, to be comparable with

9 filtering of TCGA. Characteristics of these cohorts are listed in Supplemental Table 4.

11 Immune Cell Type Deconvolution from Bulk RNA-Sequencing Data

12 The MIXTURE algorithm (Fernandez et al., 2019) builds on the nu-Support Vector Regression

13 framework used by CIBERSORT (Newman et al., 2015) for particular use with noisy tumor

14 samples. MIXTURE applies Recursive Feature Selection to make the cell type deconvolution

15 more robust to noise and collinearity, and was thus designed to improve performance on tumor 16 data.

18 We run MIXTURE using a population-based null distribution and the nu-SVM Robust RFE 19 method on the preprocessed RNA-sequencing data from both TCGA and GTEx. A signature 20 expression matrix (LM22 from Newman et al) (Newman et al., 2015) is used to determine the 21 proportion of 22 immune cell types in each sample. MIXTURE returns both relative and absolute 22 proportions of immune cells. Absolute proportions were used for all analyses of TCGA and 23 GTEx datasets. MIXTURE provides a p-value for the cell type deconvolution performed. Only 24 samples with a deconvolution $p$-value less than 0.05 were used in the final analyses, leaving 253576 patient samples remaining in TCGA and 1689 in GTEx. A further 29 TCGA patients had 26 received treatment prior to sample collection, and were removed to avoid biasing of results. 
2 Modeling the Association of Immune Cell Type with Age and Survival

3 Linear models are fit to investigate the association between the absolute proportion of each

4 immune cell type and the initial diagnosis age in TCGA. The models are fit separately for each

5 cancer type as well as jointly with cancer type and patient sex as covariates. Significance is

6 assessed using Benjamini-Hochberg FDR correction for multiple testing across all cell types

7 tested.

8

9 Higher order cell types are defined by adding together individual substituent cell type values and

10 dividing by the sum of all cell types, the result of which is used as the predictor variable in the

11 linear model (which immune subtypes correspond to which higher order cell types is shown in

12 Supplemental Table 1).

14 In addition to the linear models, we made box and violin plots of the immune cell type absolute

15 proportion by age group without additional covariate adjustment to visualize immune changes

16 with age.

18 The relationships between overall survival and immune cell types are assessed using a Cox

19 proportional hazards model fit with the survival R package Version 3.1-8. The model uses

20 months of overall survival as the outcome and includes diagnosis age and sex as covariates. It

21 is further stratified by cancer type.

22

23 GTEx data only provides the age group of each individual rather than the particular year of age

24 at the time of sample collection, so an ANOVA is performed between absolute immune cell

25 proportion and age group, including age and tissue type as covariates. 
1 Modeling the Age Associations of Normalized TCR Clonality and Number of Tumor Mutations

2 TCR clonality is assessed using miTCR (Bolotin et al., 2013) results previously published by

3 Thorsson et al., 2018 (Thorsson et al., 2018). Our previous results demonstrated decreased

4 infiltration of T cells with increasing age, so to avoid biasing our results, the Shannon Entropy is

5 multiplied by the number of unique TCR clones divided by the total number of TCR reads. We

6 then fit a linear model for the association of age with this TCR clonality measure, including

7 patient sex and cancer type as covariates. We again use a Cox Proportional hazards model to

8 assess if normalized TCR clonality is a relevant survival prognostic, using the same survival

9 function and covariates as described above.

11 To find the association of age and number of tumor mutations we downloaded the mutation

12 count provided for each sample from the GDC data portal. We log transformed the data due to

13 the skewed distribution of tumor mutation counts and fit the values to a linear model and Cox

14 proportional hazards model, using the same covariates as above.

Differential Expression Analysis with Age

17 Differential expression analyses from both TCGA and GTEx data were performed on all

18 samples from individuals of at least 30 years of age. The edgeR package Version 3.26.8 was

19 used for normalization and identifying differentially expressed genes with age. Diagnosis age

20 was modeled as a continuous variable, including cancer type as a covariate for the TCGA

21 analysis and tissue type as a covariate for the GTEx analysis. Genes were considered

22 differentially expressed below an FDR adjusted $p$-value of 0.05 . A further differential expression

23 analysis was performed on TCGA data, including mutation count as an additional covariate.

24 Finally, a differential expression analysis for diagnosis age was performed on each cancer type 25 separately that had at least 100 samples. 
1 Differential Methylation Analysis with Age

2 Merged 450k and 27k DNA methylation array data preprocessed by (Thorsson et al., 2018) was

3 downloaded from GDC at https://gdc.cancer.gov/about-data/publications/panimmune. A linear

4 model for diagnosis age was fit to data from each $\mathrm{CpG}$, including cancer type as a covariate.

5 CpG methylation was considered significantly different with age if the FDR adjusted p-value for

6 the diagnosis age term was less than 0.05. Annotations of $\mathrm{CpG}$ sites to gene promoters were

7 retrieved from the IlluminaHumanMethylation27k.db R package Version 1.4.8. The same

8 analysis was repeated, additionally including mutation count as a covariate in the model.

10 Finding Epigenetically Regulated Immune Genes

11 Genes annotated to have an association with immune function were downloaded from InnateDB

12 (Breuer et al., 2013), (Lynn et al., 2008). Differentially expressed genes were subset to those

13 overlapping with the set annotated as immune-related. These sets were then further subset to

14 those that were up-expressed with age and down-methylated, as well as those down-expressed

15 with age and up-methylated, in accordance with the canonical understanding of the effects of

16 DNA methylation on gene expression.

18 These sets were produced for both the analysis including and not including mutation count as a 19 covariate. The resulting gene sets were visualized using the STRING database (Szklarczyk et

20 al., 2019), which shows the known or predicted interactions that the corresponding proteins

21 would be expected to have.

\section{Gene Set Enrichment Analysis}

24 The fgsea R package version 1.10.1 (Sergushichev, 2016) was used to perform gene set

25 enrichment analysis from differential expression results with age from TCGA and GTEx,

26 produced as described above. GO terms were downloaded from MsigDB (Liberzon et al., 2011) 
bioRxiv preprint doi: https://doi.org/10.1101/2020.06.08.140764; this version posted June 8, 2020. The copyright holder for this preprint (which was not certified by peer review) is the author/funder, who has granted bioRxiv a license to display the preprint in perpetuity. It is made available under aCC-BY-NC 4.0 International license.

1 using the msigdbr R package Version 7.0.1. GO enrichment was compared between the data

2 sets, identifying those terms significantly up in both, up in one and down in the other, and down

3 in both with age. 


\section{Code Availability}

All analysis code is available on GitHub at: https://github.com/rossinerbe/ImmuneAgingAnalysis

\section{Acknowledgements}

The authors would like to thank Ashani T. Weeraratna for her input on aging immunity in cancer.

The authors would also like to thank Mara R. Lanis for her helpful discussions on TCR diversity and immune checkpoint genes.

The results shown here are based upon data generated by the TCGA Research Network:

https://www.cancer.gov/tcga and the The Genotype-Tissue Expression (GTEx) Project

https://www.gtexportal.org/.

\section{Declaration of Interests}

The authors declare no competing interests.

\section{References}

Alpert, A., Pickman, Y., Leipold, M., Rosenberg-Hasson, Y., Ji, X., Gaujoux, R., Rabani, H., Starosvetsky, E., Kveler, K., Schaffert, S., et al. (2019). A clinically meaningful metric of immune age derived from high-dimensional longitudinal monitoring. Nat. Med. 25, 487-495.

Asgarova, A., Asgarov, K., Godet, Y., Peixoto, P., Nadaradjane, A., Boyer-Guittaut, M., Galaine, J., Guenat, D., Mougey, V., Perrard, J., et al. (2018). PD-L1 expression is regulated by both DNA methylation and NF-kB during EMT signaling in non-small cell lung carcinoma. Oncoimmunology 7, e1423170.

Aspinall, R., and Andrew, D. (2000). Thymic involution in aging. J. Clin. Immunol. 20, 250-256.

Aw, D., Silva, A.B., and Palmer, D.B. (2007). Immunosenescence: emerging challenges for an ageing population. Immunology 120, 435-446.

Bolotin, D.A., Shugay, M., Mamedov, I.Z., Putintseva, E.V., Turchaninova, M.A., Zvyagin, I.V., Britanova, O.V., and Chudakov, D.M. (2013). MiTCR: software for T-cell receptor sequencing data analysis. Nat. Methods 10, 813-814. 
Breda, C.N. de S., Davanzo, G.G., Basso, P.J., Saraiva Câmara, N.O., and Moraes-Vieira, P.M.M. (2019). Mitochondria as central hub of the immune system. Redox Biol. 26, 101255.

Breuer, K., Foroushani, A.K., Laird, M.R., Chen, C., Sribnaia, A., Lo, R., Winsor, G.L., Hancock, R.E.W., Brinkman, F.S.L., and Lynn, D.J. (2013). InnateDB: systems biology of innate immunity and beyond--recent updates and continuing curation. Nucleic Acids Res. 41, D1228-33.

Britanova, O.V., Putintseva, E.V., Shugay, M., Merzlyak, E.M., Turchaninova, M.A., Staroverov, D.B., Bolotin, D.A., Lukyanov, S., Bogdanova, E.A., Mamedov, I.Z., et al. (2014). Age-related decrease in TCR repertoire diversity measured with deep and normalized sequence profiling. J. Immunol. 192, 2689-2698.

Cancer Genome Atlas Research Network, Kandoth, C., Schultz, N., Cherniack, A.D., Akbani, R., Liu, Y., Shen, H., Robertson, A.G., Pashtan, I., Shen, R., et al. (2013). Integrated genomic characterization of endometrial carcinoma. Nature 497, 67-73.

Chalmers, Z.R., Connelly, C.F., Fabrizio, D., Gay, L., Ali, S.M., Ennis, R., Schrock, A., Campbell, B., Shlien, A., Chmielecki, J., et al. (2017). Analysis of 100,000 human cancer genomes reveals the landscape of tumor mutational burden. Genome Med. 9, 34.

Chanmee, T., Ontong, P., Konno, K., and Itano, N. (2014). Tumor-associated macrophages as major players in the tumor microenvironment. Cancers (Basel) 6, 1670-1690.

Chiossone, L., Dumas, P.-Y., Vienne, M., and Vivier, E. (2018). Natural killer cells and other innate lymphoid cells in cancer. Nat. Rev. Immunol. 18, 671-688.

Daste, A., Domblides, C., Gross-Goupil, M., Chakiba, C., Quivy, A., Cochin, V., de Mones, E., Larmonier, N., Soubeyran, P., and Ravaud, A. (2017). Immune checkpoint inhibitors and elderly people: A review. Eur. J. Cancer 82, 155-166.

Easwaran, H., Johnstone, S.E., Van Neste, L., Ohm, J., Mosbruger, T., Wang, Q., Aryee, M.J., Joyce, P., Ahuja, N., Weisenberger, D., et al. (2012). A DNA hypermethylation module for the stem/progenitor cell signature of cancer. Genome Res. 22, 837-849.

Egorov, E.S., Kasatskaya, S.A., Zubov, V.N., Izraelson, M., Nakonechnaya, T.O., Staroverov, D.B., Angius, A., Cucca, F., Mamedov, I.Z., Rosati, E., et al. (2018). The changing landscape of naive $\mathrm{T}$ cell receptor repertoire with human aging. Front. Immunol. 9, 1618.

Elias, R., Giobbie-Hurder, A., McCleary, N.J., Ott, P., Hodi, F.S., and Rahma, O. (2018). Efficacy of PD-1 \& PD-L1 inhibitors in older adults: a meta-analysis. J. Immunother. Cancer 6, 26.

Fernandez, E., Mahmoud, Y.D., Veigas, F., Rocha, D., Balzarini, M., Lujan, H.D., Rabinovich, G.A., and Girotti, M.R. (2019). MIXTURE: an improved algorithm for immune tumor microenvironment estimation based on gene expression data. BioRxiv.

Flies, D.B., and Chen, L. (2007). The new B7s: playing a pivotal role in tumor immunity. J. Immunother. 30, 251-260.

Franceschi, C., Bonafè, M., Valensin, S., Olivieri, F., De Luca, M., Ottaviani, E., and De Benedictis, G. (2000). Inflamm-aging. An evolutionary perspective on immunosenescence. Ann. N. Y. Acad. Sci. 908, 244-254. 
Frankel, T., Lanfranca, M.P., and Zou, W. (2017). The role of tumor microenvironment in cancer immunotherapy. Adv. Exp. Med. Biol. 1036, 51-64.

Freeman, A.J., Vervoort, S.J., Ramsbottom, K.M., Kelly, M.J., Michie, J., Pijpers, L., Johnstone, R.W., Kearney, C.J., and Oliaro, J. (2019). Natural Killer Cells Suppress T Cell-Associated Tumor Immune Evasion. Cell Rep. 28, 2784-2794.e5.

Fulop, T., Larbi, A., Dupuis, G., Le Page, A., Frost, E.H., Cohen, A.A., Witkowski, J.M., and Franceschi, C. (2017). Immunosenescence and Inflamm-Aging As Two Sides of the Same Coin: Friends or Foes? Front. Immunol. 8, 1960.

Gardner, I.D. (1980). The effect of aging on susceptibility to infection. Rev. Infect. Dis. 2, 801810.

Goodman, A.M., Kato, S., Bazhenova, L., Patel, S.P., Frampton, G.M., Miller, V., Stephens, P.J., Daniels, G.A., and Kurzrock, R. (2017). Tumor mutational burden as an independent predictor of response to immunotherapy in diverse cancers. Mol. Cancer Ther. 16, 2598-2608.

Gounder, S.S., Abdullah, B.J.J., Radzuanb, N.E.I.B.M., Zain, F.D.B.M., Sait, N.B.M., Chua, C., and Subramani, B. (2018). Effect of aging on NK cell population and their proliferation at ex vivo culture condition. Anal Cell Pathol (Amst) 2018, 7871814.

GTEx Consortium, Laboratory, Data Analysis \&Coordinating Center (LDACC)—Analysis Working Group, Statistical Methods groups-Analysis Working Group, Enhancing GTEx (eGTEx) groups, NIH Common Fund, NIH/NCI, NIH/NHGRI, NIH/NIMH, NIH/NIDA, Biospecimen Collection Source Site-NDRI, et al. (2017). Genetic effects on gene expression across human tissues. Nature 550, 204-213.

Hanahan, D., and Weinberg, R.A. (2011). Hallmarks of cancer: the next generation. Cell 144, 646-674.

Hartley, G.P., Chow, L., Ammons, D.T., Wheat, W.H., and Dow, S.W. (2018). Programmed Cell Death Ligand 1 (PD-L1) Signaling Regulates Macrophage Proliferation and Activation. Cancer Immunol. Res. 6, 1260-1273.

Horvath, S. (2013). DNA methylation age of human tissues and cell types. Genome Biol. 14, R115.

Hulstaert, F., Hannet, I., Deneys, V., Munhyeshuli, V., Reichert, T., De Bruyere, M., and Strauss, K. (1994). Age-related changes in human blood lymphocyte subpopulations. II. Varying kinetics of percentage and absolute count measurements. Clin. Immunol. Immunopathol. 70, 152-158.

Hurez, V., Padrón, Á., Svatek, R.S., and Curiel, T.J. (2018). Considerations for successful cancer immunotherapy in aged hosts. Exp. Gerontol. 107, 27-36.

Jain, V., Hwang, W.-T., Venigalla, S., Nead, K.T., Lukens, J.N., Mitchell, T.C., and Shabason, J.E. (2019). Association of Age with Efficacy of Immunotherapy in Metastatic Melanoma. Oncologist.

Klümper, N., Ralser, D.J., Bawden, E.G., Landsberg, J., Zarbl, R., Kristiansen, G., Toma, M., Ritter, M., Hölzel, M., Ellinger, J., et al. (2020). LAG3 (LAG-3, CD223) DNA methylation 
correlates with LAG3 expression by tumor and immune cells, immune cell infiltration, and overall survival in clear cell renal cell carcinoma. J. Immunother. Cancer 8.

Kovtonyuk, L.V., Fritsch, K., Feng, X., Manz, M.G., and Takizawa, H. (2016). Inflamm-Aging of Hematopoiesis, Hematopoietic Stem Cells, and the Bone Marrow Microenvironment. Front. Immunol. 7, 502.

Kugel, C.H., Douglass, S.M., Webster, M.R., Kaur, A., Liu, Q., Yin, X., Weiss, S.A., Darvishian, F., Al-Rohil, R.N., Ndoye, A., et al. (2018). Age Correlates with Response to Anti-PD1, Reflecting Age-Related Differences in Intratumoral Effector and Regulatory T-Cell Populations. Clin. Cancer Res. 24, 5347-5356.

Lee, C., Jeong, H., Bae, Y., Shin, K., Kang, S., Kim, H., Oh, J., and Bae, H. (2019). Targeting of M2-like tumor-associated macrophages with a melittin-based pro-apoptotic peptide. J. Immunother. Cancer 7, 147.

Lee, K.-A., Shin, K.-S., Kim, G.-Y., Song, Y.C., Bae, E.-A., Kim, I.-K., Koh, C.-H., and Kang, C.$Y$. (2016). Characterization of age-associated exhausted $C D 8^{+} \mathrm{T}$ cells defined by increased expression of Tim-3 and PD-1. Aging Cell 15, 291-300.

Liberzon, A., Subramanian, A., Pinchback, R., Thorvaldsdóttir, H., Tamayo, P., and Mesirov, J.P. (2011). Molecular signatures database (MSigDB) 3.0. Bioinformatics 27, 1739-1740.

Long, L., Zhang, X., Chen, F., Pan, Q., Phiphatwatchara, P., Zeng, Y., and Chen, H. (2018). The promising immune checkpoint LAG-3: from tumor microenvironment to cancer immunotherapy. Genes Cancer 9, 176-189.

Lu, C., Rong, D., Zhang, B., Zheng, W., Wang, X., Chen, Z., and Tang, W. (2019). Current perspectives on the immunosuppressive tumor microenvironment in hepatocellular carcinoma: challenges and opportunities. Mol. Cancer 18, 130.

Lynn, D.J., Winsor, G.L., Chan, C., Richard, N., Laird, M.R., Barsky, A., Gardy, J.L., Roche, F.M., Chan, T.H.W., Shah, N., et al. (2008). InnateDB: facilitating systems-level analyses of the mammalian innate immune response. Mol. Syst. Biol. 4, 218.

Mahbub, S., Deburghgraeve, C.R., and Kovacs, E.J. (2012). Advanced age impairs macrophage polarization. J. Interferon Cytokine Res. 32, 18-26.

McNeel, D.G. (2016). TCR diversity - a universal cancer immunotherapy biomarker? J. Immunother. Cancer 4, 69.

Micevic, G., Thakral, D., McGeary, M., and Bosenberg, M.W. (2019). PD-L1 methylation regulates PD-L1 expression and is associated with melanoma survival. Pigment Cell Melanoma Res. 32, 435-440.

Newman, A.M., Liu, C.L., Green, M.R., Gentles, A.J., Feng, W., Xu, Y., Hoang, C.D., Diehn, M., and Alizadeh, A.A. (2015). Robust enumeration of cell subsets from tissue expression profiles. Nat. Methods 12, 453-457.

Normanno, N., De Luca, A., Bianco, C., Strizzi, L., Mancino, M., Maiello, M.R., Carotenuto, A., De Feo, G., Caponigro, F., and Salomon, D.S. (2006). Epidermal growth factor receptor (EGFR) signaling in cancer. Gene 366, 2-16. 
Ohm, J.E., and Baylin, S.B. (2007). Stem cell chromatin patterns: an instructive mechanism for DNA hypermethylation? Cell Cycle 6, 1040-1043.

Pandini, G., Vigneri, R., Costantino, A., Frasca, F., Ippolito, A., Fujita-Yamaguchi, Y., Siddle, K., Goldfine, I.D., and Belfiore, A. (1999). Insulin and insulin-like growth factor-I (IGF-I) receptor overexpression in breast cancers leads to insulin/IGF-I hybrid receptor overexpression: evidence for a second mechanism of IGF-I signaling. Clin. Cancer Res. 5, 1935-1944.

Poh, A.R., and Ernst, M. (2018). Targeting macrophages in cancer: from bench to bedside. Front. Oncol. 8, 49.

Qing, T., Mohsen, H., Marczyk, M., Ye, Y., O’Meara, T., Zhao, H., Townsend, J.P., Gerstein, M., Hatzis, C., Kluger, Y., et al. (2020). Germline variant burden in cancer genes correlates with age at diagnosis and somatic mutation burden. Nat. Commun. 11, 2438.

Rozenblatt-Rosen, O., Regev, A., Oberdoerffer, P., Nawy, T., Hupalowska, A., Rood, J.E., Ashenberg, O., Cerami, E., Coffey, R.J., Demir, E., et al. (2020). The Human Tumor Atlas Network: Charting Tumor Transitions across Space and Time at Single-Cell Resolution. Cell 181, 236-249.

Schlesinger, Y., Straussman, R., Keshet, I., Farkash, S., Hecht, M., Zimmerman, J., Eden, E., Yakhini, Z., Ben-Shushan, E., Reubinoff, B.E., et al. (2007). Polycomb-mediated methylation on Lys27 of histone H3 pre-marks genes for de novo methylation in cancer. Nat. Genet. 39, 232236.

Sergushichev, A. (2016). An algorithm for fast preranked gene set enrichment analysis using cumulative statistic calculation. BioRxiv.

Shimasaki, N., Jain, A., and Campana, D. (2020). NK cells for cancer immunotherapy. Nat. Rev. Drug Discov. 19, 200-218.

Smith, L.K., Boukhaled, G.M., Condotta, S.A., Mazouz, S., Guthmiller, J.J., Vijay, R., Butler, N.S., Bruneau, J., Shoukry, N.H., Krawczyk, C.M., et al. (2018). Interleukin-10 Directly Inhibits CD8+ T Cell Function by Enhancing N-Glycan Branching to Decrease Antigen Sensitivity. Immunity 48, 299-312.e5.

Solana, R., and Mariani, E. (2000). NK and NK/T cells in human senescence. Vaccine 18, 1613-1620.

Soufla, G., Sifakis, S., Baritaki, S., Zafiropoulos, A., Koumantakis, E., and Spandidos, D.A. (2005). VEGF, FGF2, TGFB1 and TGFBR1 mRNA expression levels correlate with the malignant transformation of the uterine cervix. Cancer Lett. 221, 105-118.

Szklarczyk, D., Gable, A.L., Lyon, D., Junge, A., Wyder, S., Huerta-Cepas, J., Simonovic, M., Doncheva, N.T., Morris, J.H., Bork, P., et al. (2019). STRING v11: protein-protein association networks with increased coverage, supporting functional discovery in genome-wide experimental datasets. Nucleic Acids Res. 47, D607-D613.

Taube, J.M. (2014). Unleashing the immune system: PD-1 and PD-Ls in the pre-treatment tumor microenvironment and correlation with response to PD-1/PD-L1 blockade.

Oncoimmunology 3, e963413. 
Thorsson, V., Gibbs, D.L., Brown, S.D., Wolf, D., Bortone, D.S., Ou Yang, T.-H., Porta-Pardo, E., Gao, G.F., Plaisier, C.L., Eddy, J.A., et al. (2018). The immune landscape of cancer. Immunity 48, 812-830.e14.

Tomasetti, C., Li, L., and Vogelstein, B. (2017). Stem cell divisions, somatic mutations, cancer etiology, and cancer prevention. Science 355, 1330-1334.

Velghe, A.I., Van Cauwenberghe, S., Polyansky, A.A., Chand, D., Montano-Almendras, C.P., Charni, S., Hallberg, B., Essaghir, A., and Demoulin, J.B. (2014). PDGFRA alterations in cancer: characterization of a gain-of-function V536E transmembrane mutant as well as loss-offunction and passenger mutations. Oncogene 33, 2568-2576.

Weinberg, S.E., Sena, L.A., and Chandel, N.S. (2015). Mitochondria in the regulation of innate and adaptive immunity. Immunity 42, 406-417.

Widschwendter, M., Fiegl, H., Egle, D., Mueller-Holzner, E., Spizzo, G., Marth, C., Weisenberger, D.J., Campan, M., Young, J., Jacobs, I., et al. (2007). Epigenetic stem cell signature in cancer. Nat. Genet. 39, 157-158.

Wolf, Y., Anderson, A.C., and Kuchroo, V.K. (2020). TIM3 comes of age as an inhibitory receptor. Nat. Rev. Immunol. 20, 173-185.

Yager, E.J., Ahmed, M., Lanzer, K., Randall, T.D., Woodland, D.L., and Blackman, M.A. (2008). Age-associated decline in $\mathrm{T}$ cell repertoire diversity leads to holes in the repertoire and impaired immunity to influenza virus. J. Exp. Med. 205, 711-723.

Yang, Z.-Z., Kim, H.J., Villasboas, J.C., Chen, Y.-P., Price-Troska, T., Jalali, S., Wilson, M., Novak, A.J., and Ansell, S.M. (2017). Expression of LAG-3 defines exhaustion of intratumoral PD-1+ T cells and correlates with poor outcome in follicular lymphoma. Oncotarget 8, 6142561439.

Yarchoan, M., Hopkins, A., and Jaffee, E.M. (2017). Tumor Mutational Burden and Response Rate to PD-1 Inhibition. N. Engl. J. Med. 377, 2500-2501. 
bioRxiv preprint doi: https://doi.org/10.1101/2020.06.08.140764; this version posted June 8, 2020. The copyright holder for this preprint (which was not certified by peer review) is the author/funder, who has granted bioRxiv a license to display the preprint in perpetuity. It is made available under aCC-BY-NC 4.0 International license.

\section{Figures}

Figure 1 - Macrophages increase and T cells decrease with age in the ITME 
bioRxiv preprint doi: https://doi.org/10.1101/2020.06.08.140764; this version posted June 8, 2020. The copyright holder for this preprint (which was not certified by peer review) is the author/funder, who has granted bioRxiv a license to display the preprint in perpetuity. It is made available under aCC-BY-NC 4.0 International license.

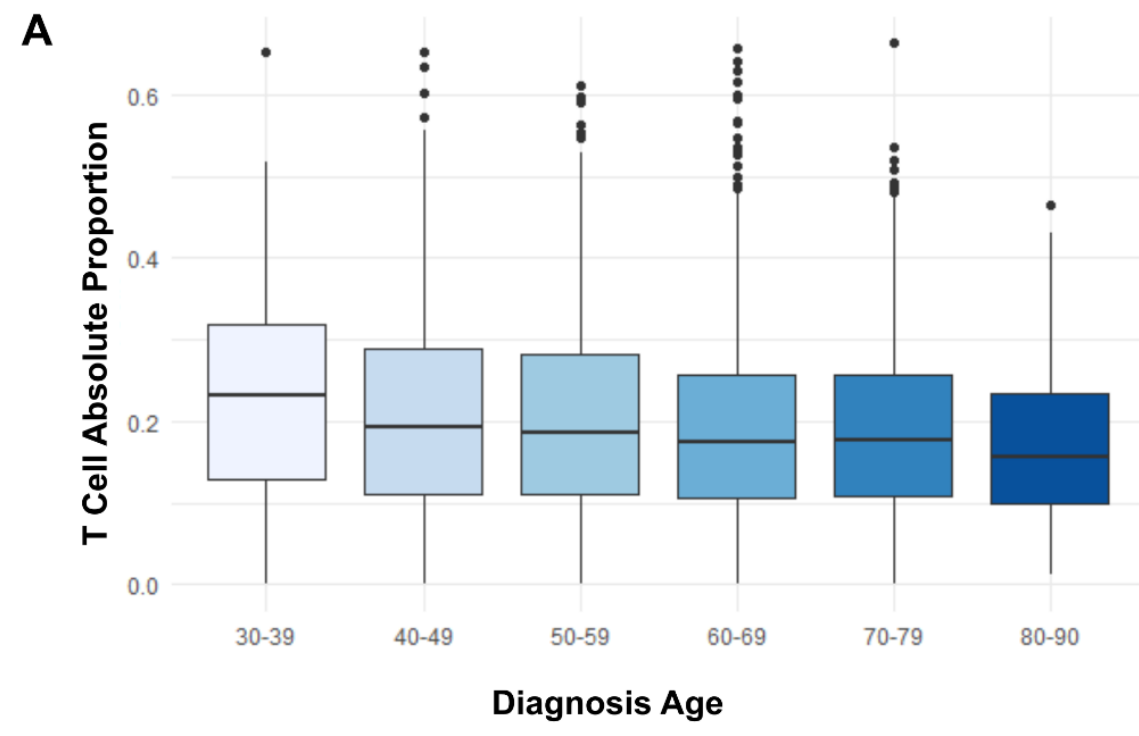

B
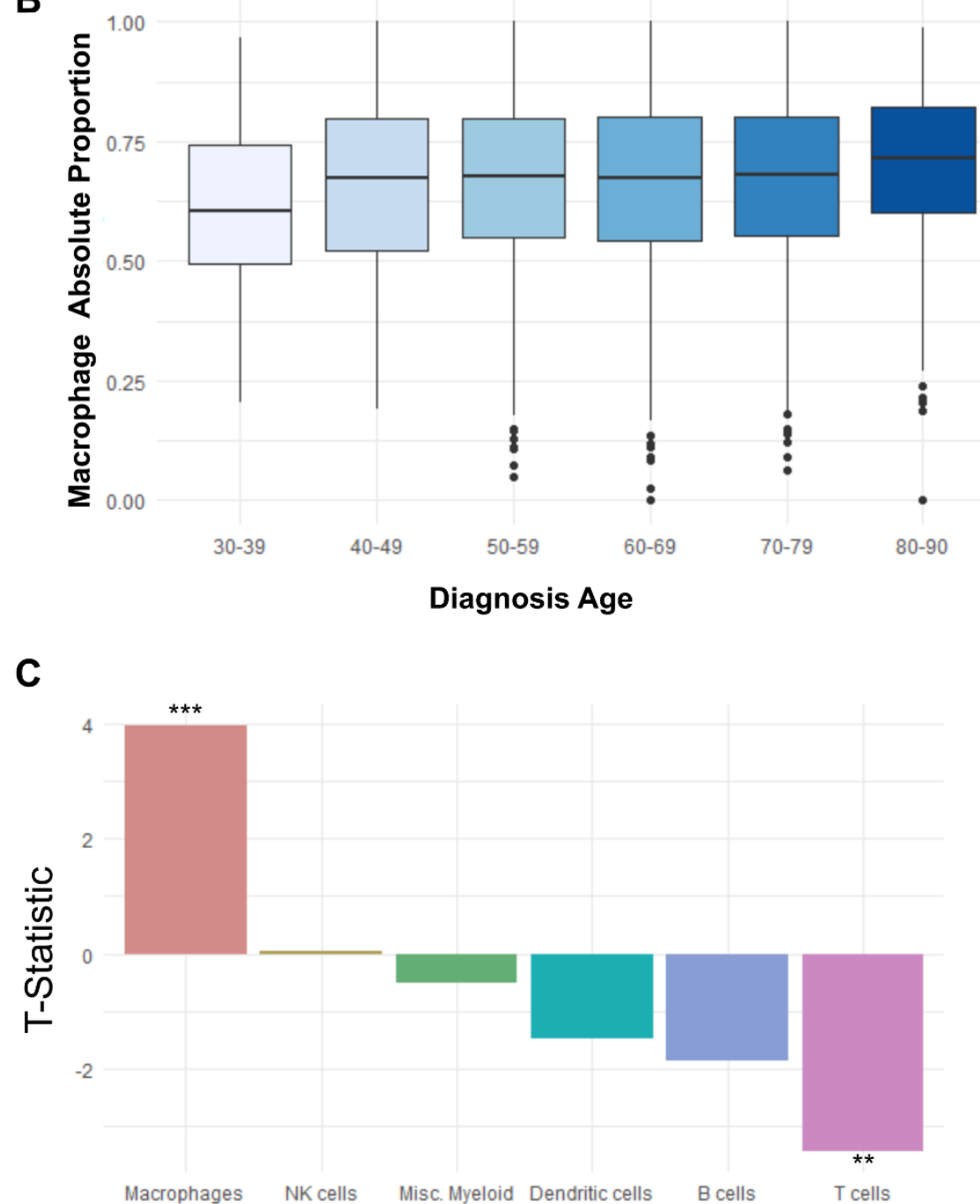

Cell Type 
bioRxiv preprint doi: https://doi.org/10.1101/2020.06.08.140764; this version posted June 8, 2020. The copyright holder for this preprint (which was not certified by peer review) is the author/funder, who has granted bioRxiv a license to display the preprint in perpetuity. It is made available under aCC-BY-NC 4.0 International license.

A Boxplots representing the pan-cancer change in T cell absolute proportion across different age groups from TCGA. A significant downward trend is observed based on a covariateadjusted linear model $B$ Boxplots representing the change in macrophage absolute proportion across age groups in TCGA. A significant upward trend is observed based on a covariateadjusted linear model $\mathbf{C}$ Barplot of the t-statistics for the diagnosis age term of the linear models fit to each immune cell type from TCGA, including sex and cancer type as covariates. ** indicates an adjusted $p$-value less than 0.01 and ${ }^{* * *}$ indicates less than 0.001 .

Figure 2 - Immune infiltration shifts with age and prognostics 
A
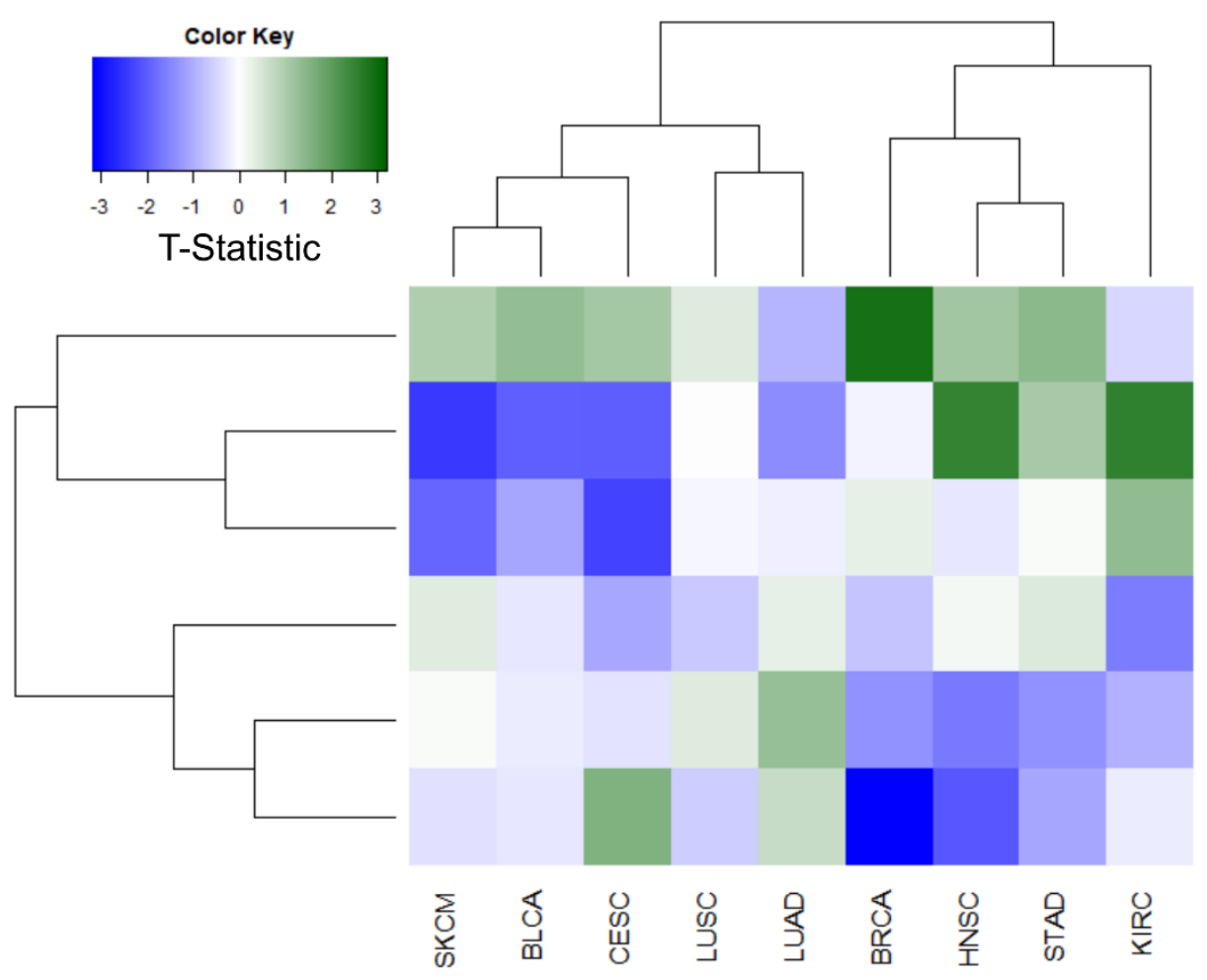

Macrophages

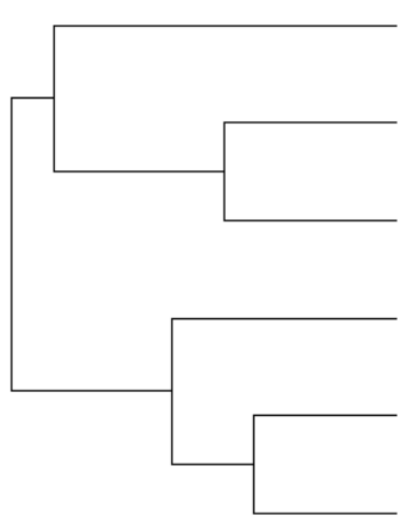

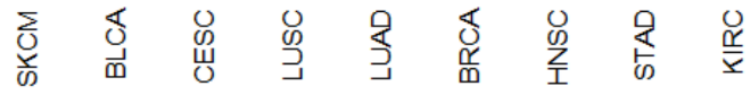

NK cells

Misc. Myeloid

Dendritic Cells

B cells

T cells

B

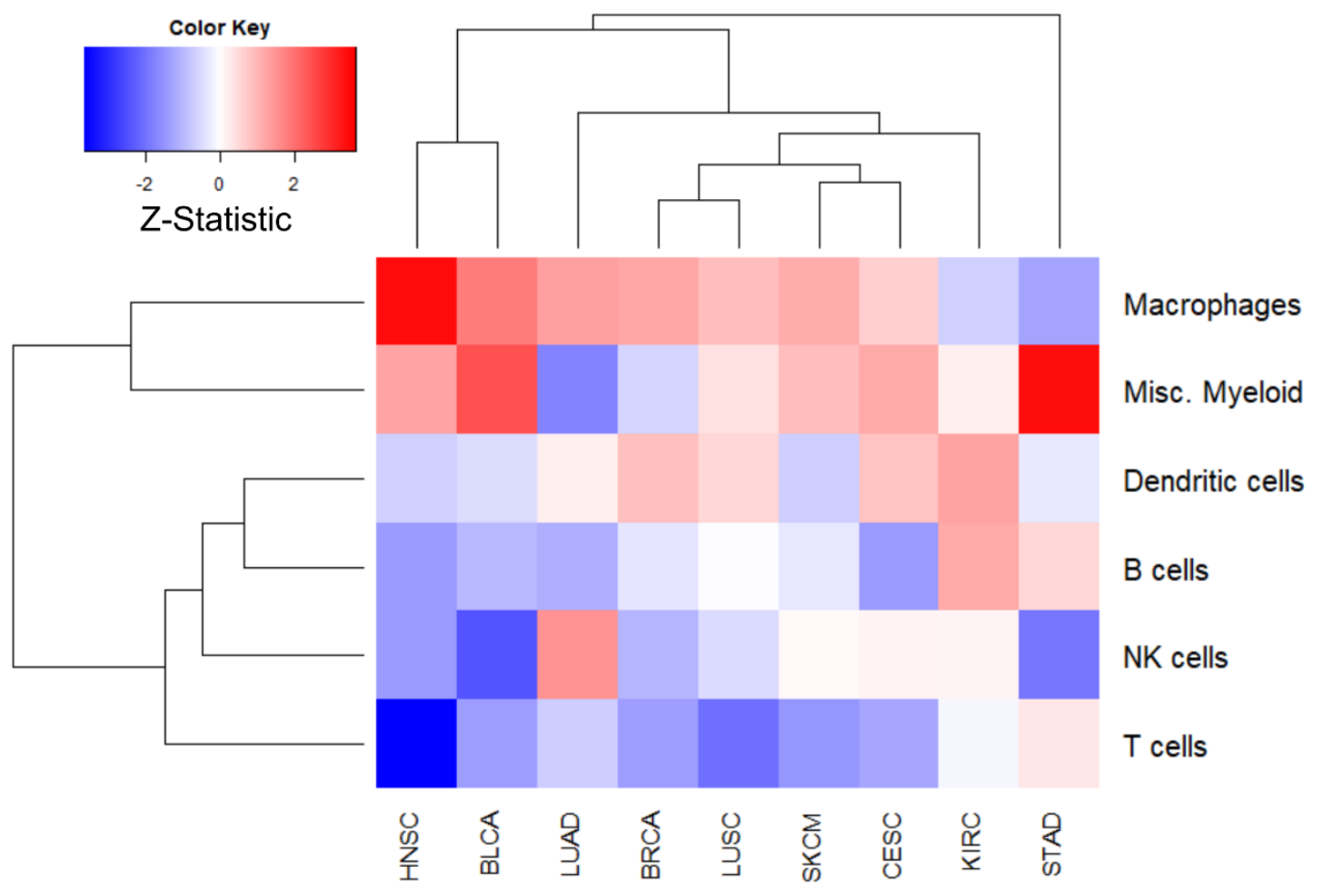


Age and survival association of immune cell types within cancer types. A Heatmap of the Tstatistics from the covariate-adjusted linear model fit for the association of each immune cell type to age. Each square represents the significance of the diagnosis age term within a linear model for the labeled immune cell type, plotted for each cancer type in TCGA with at least 100 patients after filtering for significant immune deconvolution results. We observe that macrophages generally increase in abundance with age, while T cells and B cells generally decrease in abundance. B Heatmap of the covariate-adjusted Cox proportional hazards where each square represents the z-statistic for the survival prognostic of each immune cell type within each cancer type in TCGA with at least 100 patients after filtering. We observe that macrophages and other Myeloid cells are generally poor prognostics and that $\mathrm{T}$ cells are generally good prognostics. 
Figure 3 - Immune changes with age in healthy tissues

A

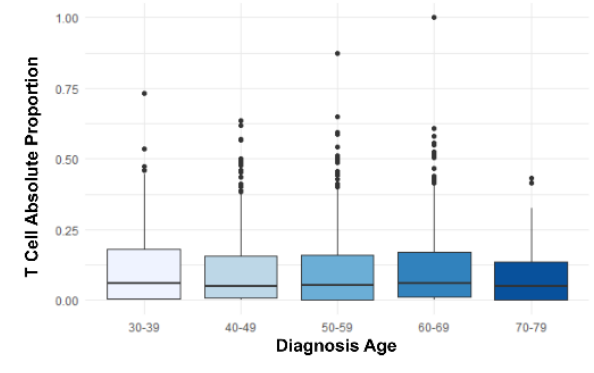

C

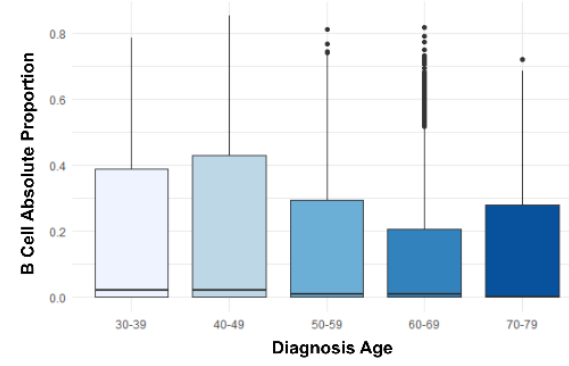

B

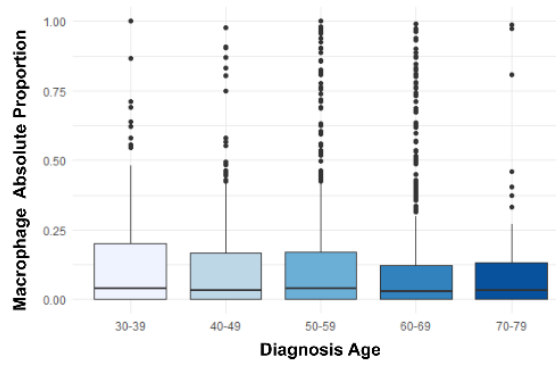

D

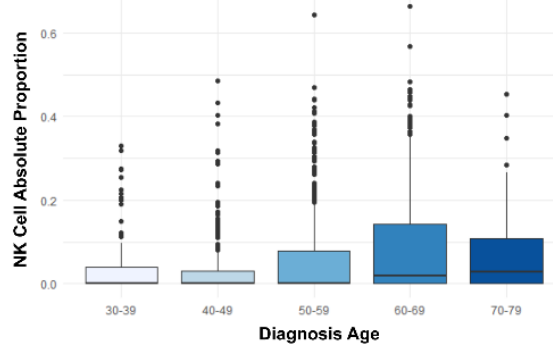

E

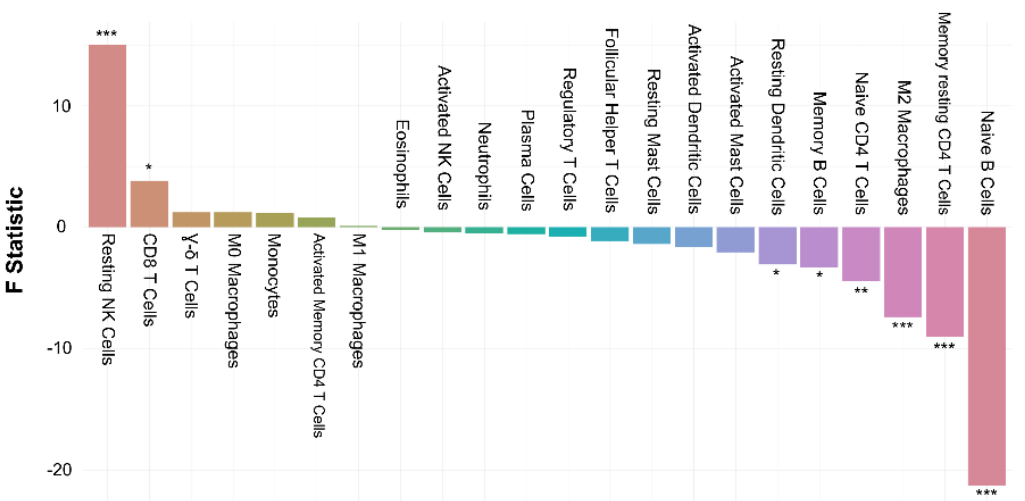

Cell Type

F

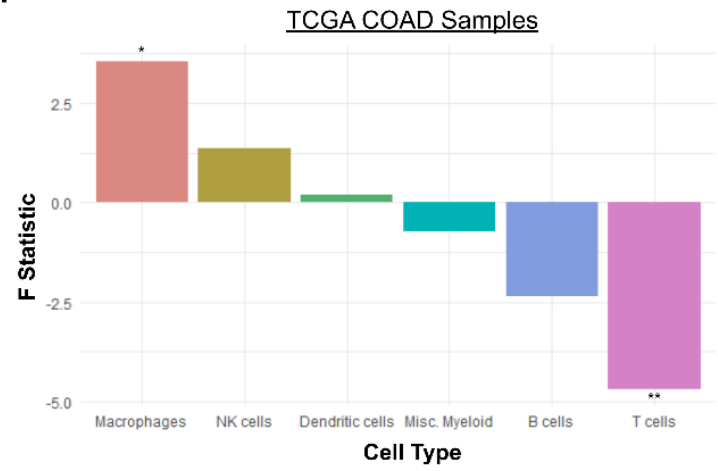

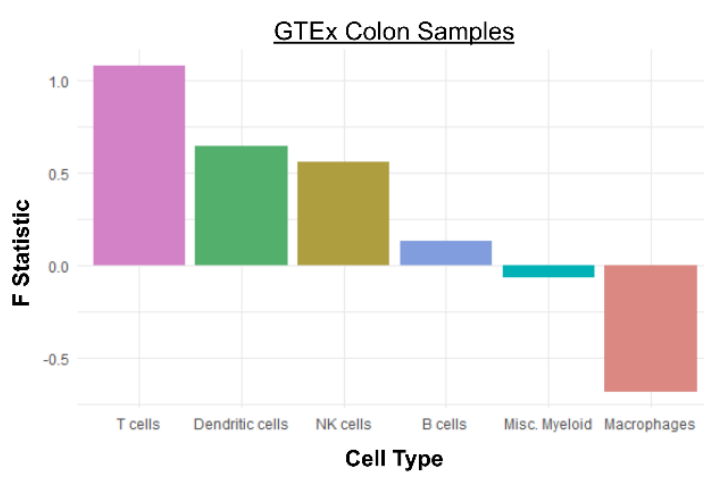

GTEx immune cell changes with age. A-D Immune abundance with age in GTEx tissues for T cells, Macrophages, B cells, and NK cells, respectively. E Age associations of 22 immune subtypes in GTEx tissues. Plotted by the F statistic for an ANOVA across age groups. $\mathbf{F}$ 
Comparison of $F$ statistics for ANOVA across age groups for TCGA Colon Adenocarcinomas (COAD) against GTEx Colon samples. Opposite trends with age are observed for macrophages and $T$ cells in this tissue. * indicates a p-value less than 0.05 and ${ }^{* *} 0.01$.

Figure 4 - Corresponding methylation and expression changes with age

A

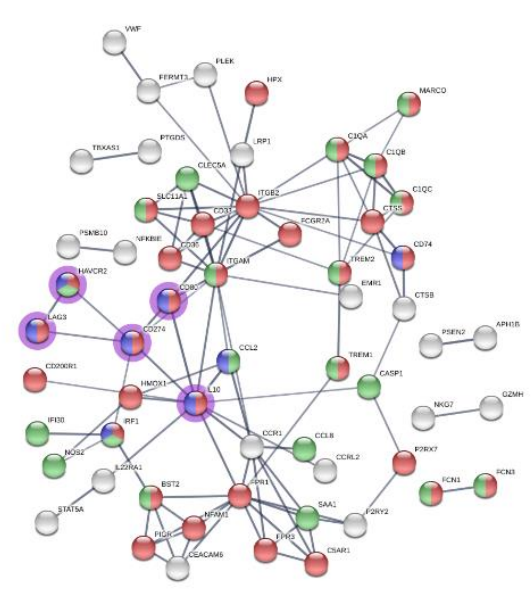

C

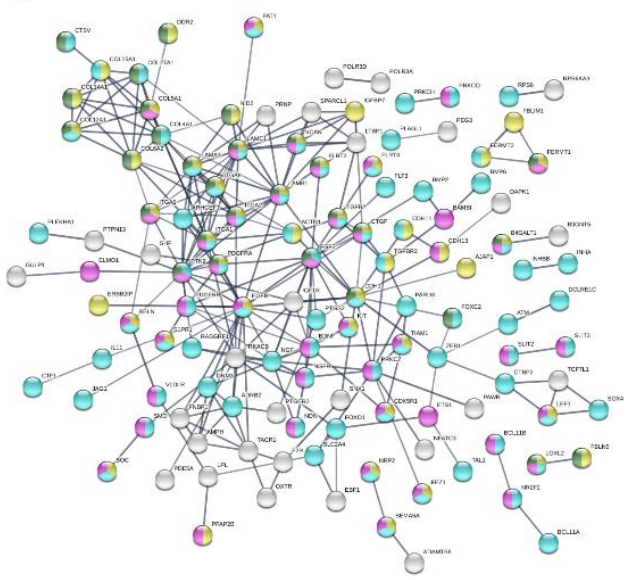

B

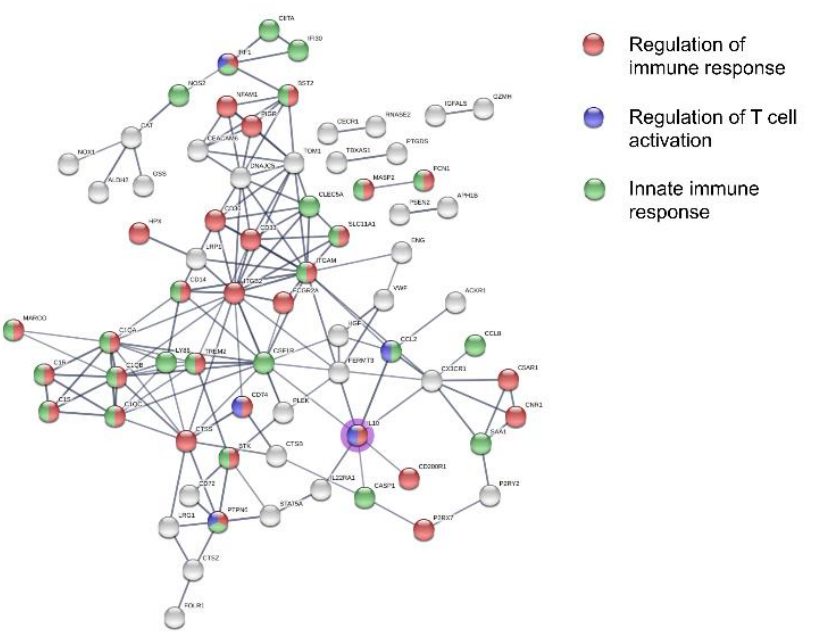

D

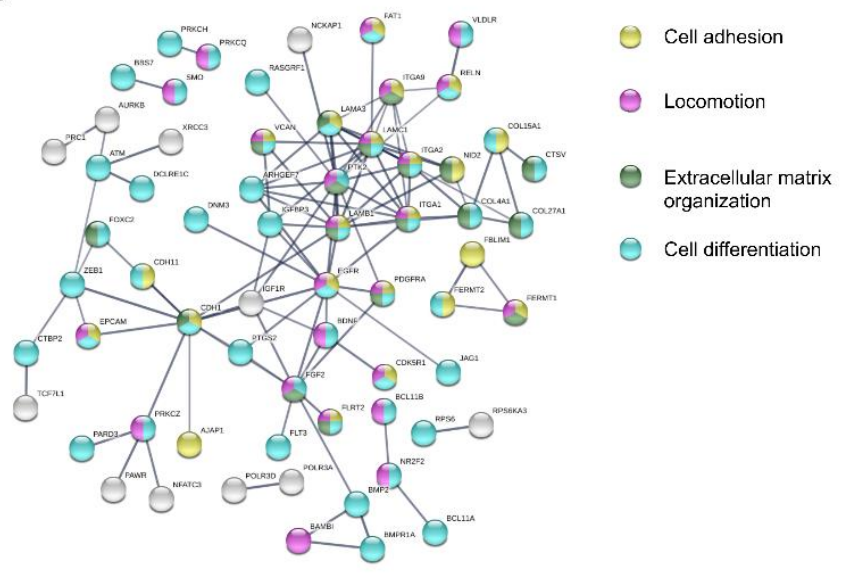

STRING Database queries for all genes with significantly $\mathbf{A}$ increased expression and decreased methylation with age, where genes that have previously been strongly associated with decreased T cell cytotoxicity have a purple halo $\mathbf{B}$ increased expression and decreased methylation with age, adjusting for tumor mutation count, where genes that have previously been strongly associated with decreased $\mathrm{T}$ cell cytotoxicity have a purple halo $\mathbf{C}$ decreased expression and increased methylation with age, D decreased expression and increased methylation with age, adjusting for tumor mutation count. 
Table 1

\begin{tabular}{|l|l|l|l|l|l|}
\hline & \multicolumn{1}{|c|}{ Estimate } & \multicolumn{1}{|c|}{$\begin{array}{c}\text { Standard } \\
\text { Error }\end{array}$} & t-value & p-value & q-value \\
\hline T cells & -0.0006 & 0.000175 & -3.44233 & 0.000585 & 0.001754 \\
\hline Macrophages & 0.001075 & 0.000271 & 3.967909 & $7.42 \mathrm{E}-05$ & 0.000445 \\
\hline B cells & -0.00028 & 0.000149 & -1.87737 & 0.060566 & 0.121131 \\
\hline NK cells & $3.82 \mathrm{E}-06$ & $6.89 \mathrm{E}-05$ & 0.055398 & 0.955826 & 0.955826 \\
\hline $\begin{array}{l}\text { Dendritic } \\
\text { cells }\end{array}$ & -0.00015 & $9.77 \mathrm{E}-05$ & -1.49017 & 0.136286 & 0.204429 \\
\hline Misc. Myeloid & $-4.97 \mathrm{E}-05$ & $9.92 \mathrm{E}-05$ & -0.50055 & 0.616722 & 0.740066 \\
\hline
\end{tabular}

Coefficients, statistics, p, and q-values for the diagnosis age term in the linear model fit for each immune cell type. Cancer type and sex were included as covariates for each of these models.

Table 2

\begin{tabular}{|l|l|l|l|l|l|}
\hline & Coefficient & \multicolumn{1}{|c|}{$\operatorname{exp(Coef.)}$} & \multicolumn{1}{|c|}{ z-statistic } & p-value & q-value \\
\hline T cells & -1.25217 & 0.285882 & -3.9169 & $8.97 \mathrm{E}-05$ & 0.000538 \\
\hline Macrophages & 0.694023 & 2.001752 & 3.460928 & 0.000538 & 0.001615 \\
\hline B cells & -0.52134 & 0.593723 & -1.43318 & 0.151808 & 0.227711 \\
\hline NK cells & -1.99242 & 0.136365 & -2.48022 & 0.01313 & 0.026261 \\
\hline $\begin{array}{l}\text { Dendritic } \\
\text { cells }\end{array}$ & 0.18663 & 1.205181 & 0.368952 & 0.712164 & 0.712164 \\
\hline Misc. Myeloid & 0.516746 & 1.676563 & 0.99876 & 0.317911 & 0.381493 \\
\hline
\end{tabular}

Coefficients, statistics, $p$, and q-values for each immune cell term in a Cox proportional hazards model fit to predict overall patient survival, including diagnosis age, sex, cancer type, and number of years smoked as covariates. 
Table 3

\begin{tabular}{|l|l|l|l|l|}
\hline & & Coefficient & Statistic & p-value \\
\hline \multirow{2}{*}{ TCR Clonality } & Diagnosis Age & -0.00509 & -5.6694 & $1.48 \mathrm{E}-08$ \\
\cline { 2 - 5 } & Cox Hazards & -0.08812 & -4.14902 & $3.34 \mathrm{E}-05$ \\
\hline $\begin{array}{l}\text { Log Tumor } \\
\text { Mutational } \\
\text { Murden }\end{array}$ & Diagnosis Age & 0.0102449 & 13.454 & $6.88 \mathrm{E}-41$ \\
& & & & \\
\cline { 2 - 5 } & Cox Hazards & -0.053340 & -2.542 & 0.011 \\
\hline
\end{tabular}

Coefficients, statistics, and p-values for the age term of the linear model for both TCR clonality and number of tumor mutations in TCGA data, with cancer type included as a covariate. These values are also listed for the TCR clonality term and the log number of tumor mutations term from a Cox proportional hazards model fit to overall survival with diagnosis age and cancer type included as covariates. 
Table 4

\begin{tabular}{|l|l|l|l|l|l|}
\hline & Gene & LogFC & t-statistic & p-value & q-value \\
\hline \multirow{4}{*}{$\begin{array}{c}\text { Age + } \\
\text { Cancer Type } \\
\text { Adjusted }\end{array}$} & CD80 & 0.00361 & 3.002352 & 0.002686 & 0.011553 \\
\cline { 2 - 6 } & CD274 & 0.003053 & 2.612337 & 0.009007 & 0.029899 \\
\cline { 2 - 6 } & CD86 & 0.002146 & 2.130932 & 0.03312 & 0.083376 \\
\cline { 2 - 6 } & CTLA4 & -0.00084 & -0.5747 & 0.56551 & 0.69286 \\
\cline { 2 - 6 }$+\begin{array}{c}+ \\
\text { Mutational } \\
\text { Bdjusted }\end{array}$ & PDCD1 & -0.00062 & -0.42127 & 0.67357 & 0.778683 \\
\cline { 2 - 6 } & CD86 & 0.002983 & -0.90527 & 2.38488 & 0.017105 \\
\cline { 2 - 6 } & CD274 & 0.002127 & 2.9119 & 2.035515 & 0.041828 \\
\cline { 2 - 6 } & CTLA4 & -0.00204 & 0.12074 & -1.34813 & 0.177652 \\
\cline { 2 - 6 } & PDCD1 & -0.002 & 0.334003 & -1.30557 & 0.191731 \\
\hline \multirow{4}{*}{} & & & & & \\
\hline
\end{tabular}

Differential expression results for immune checkpoint genes involved in currently available ICB immunotherapies. The results are shown for the association with age, both including tumor mutational burden as a covariate and without. 


\section{Supplemental Figures}

\section{Supplemental Figure 1}

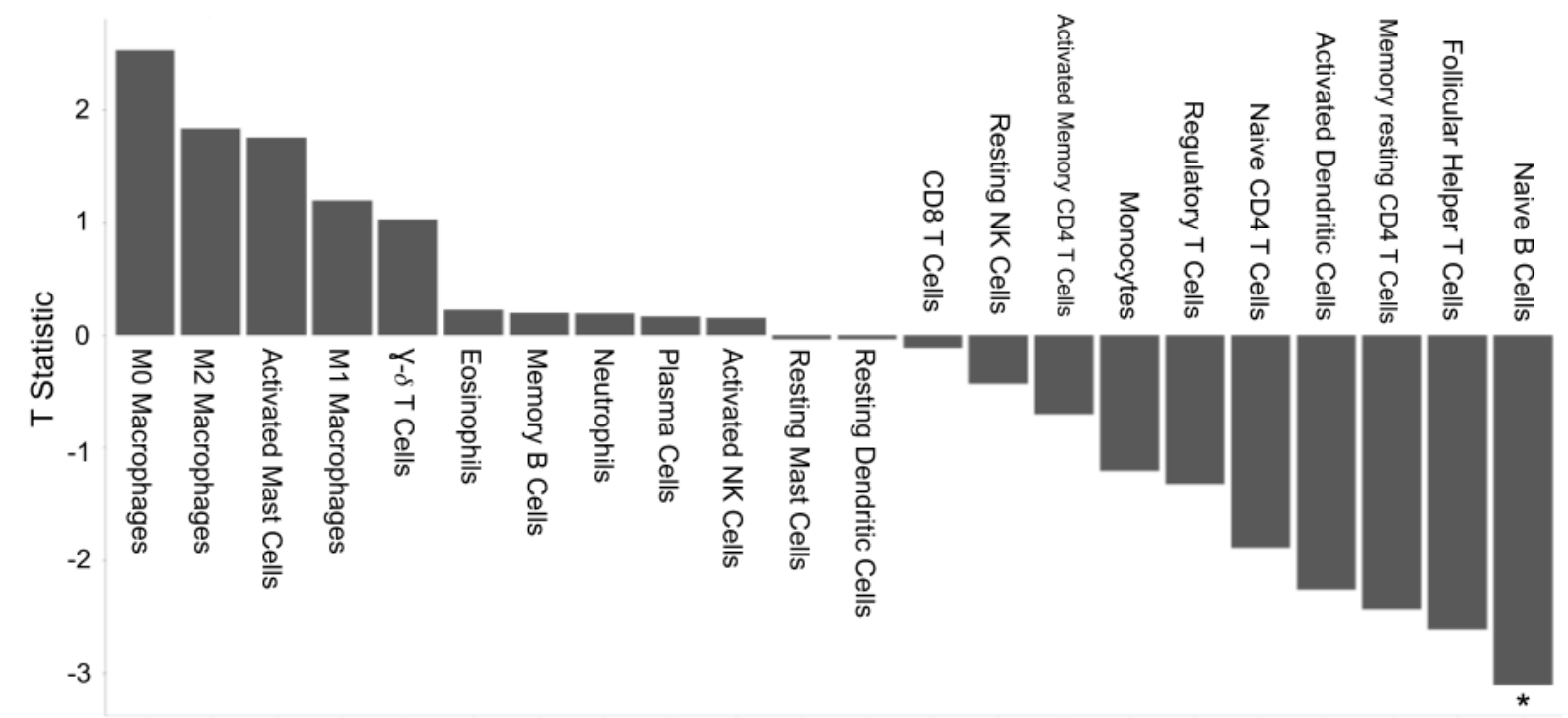

Cell Type

T statistics for the linear association of 22 immune cell subtypes with age pan-cancer within TCGA data. Naive B cells statistically significantly decrease with age. * indicates q-value less than 0.05 . 
Supplemental Figure 2

A

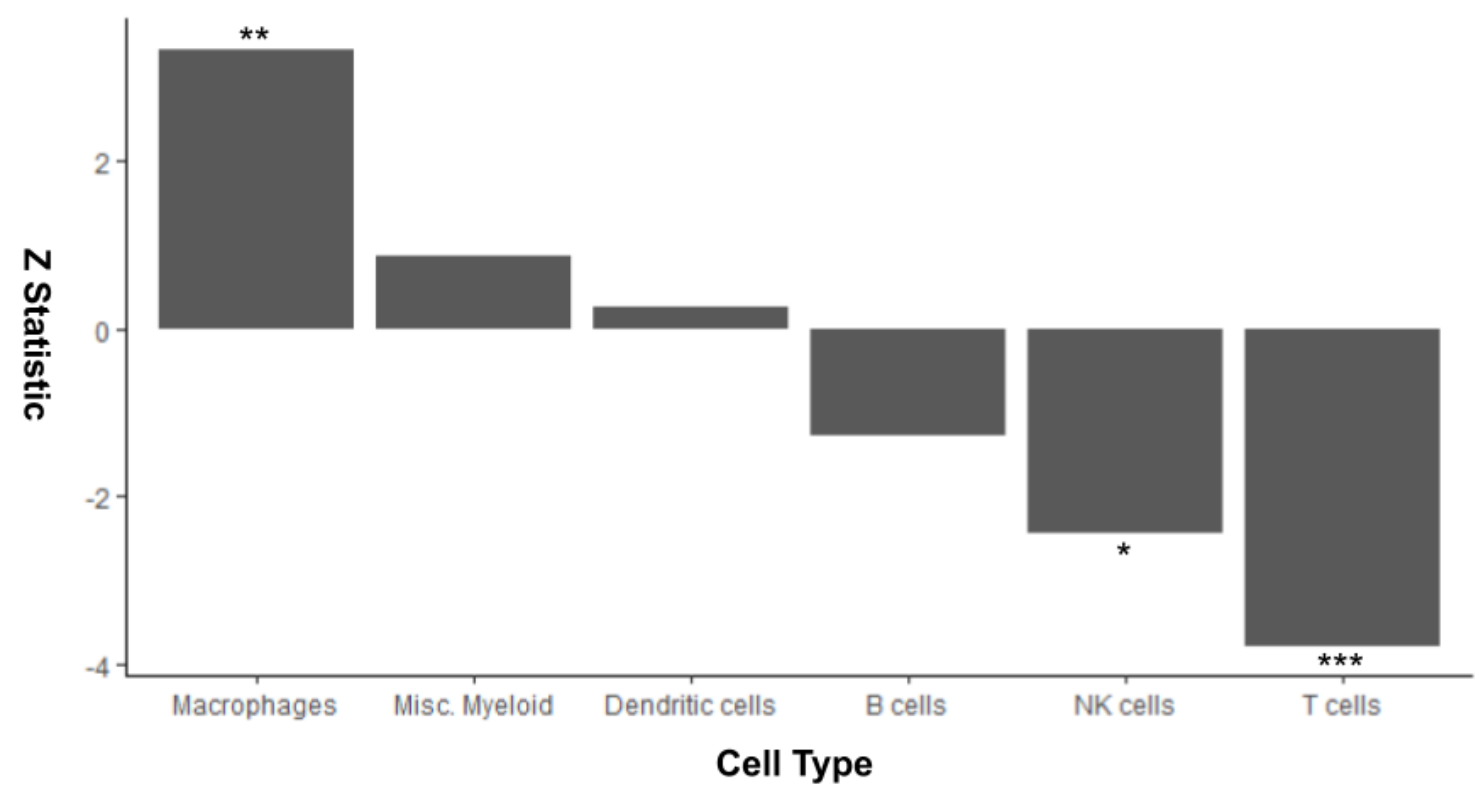

B

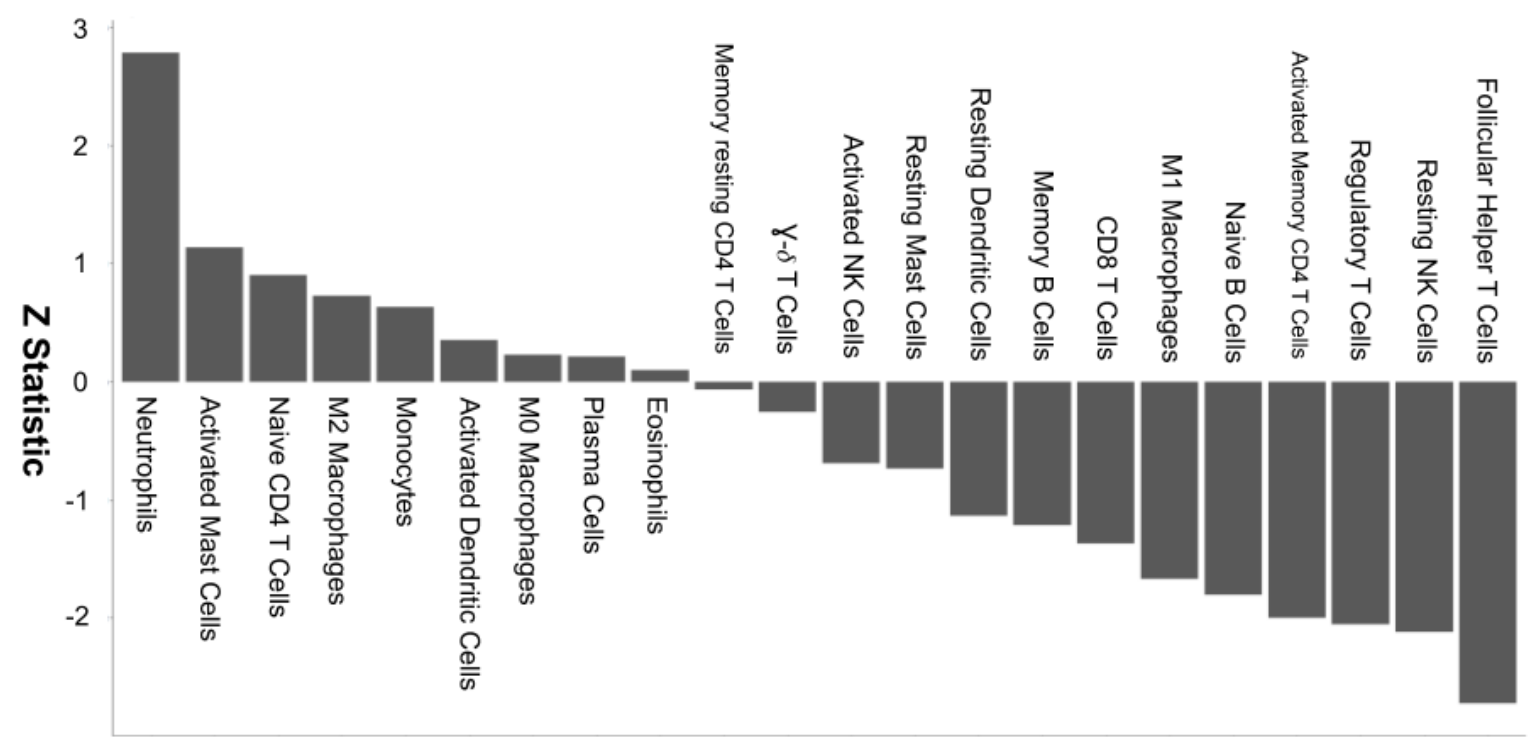

Cell Type

A Z-statistics for the survival associations of immune cell types pan-cancer as evaluated by a Cox proportional hazards model, including age, sex, cancer type, and smoking years as covariates. B Z-statistics for the survival associations of 22 immune cell subtypes as evaluated by a Cox proportional hazards model for overall patient survival pan-cancer including age, sex, cancer type, and smoking years as covariates. * indicates a p-value less than $0.05,{ }^{* *} 0.01$, and ${ }^{\star * *} 0.001$. 
Supplemental Figure 3

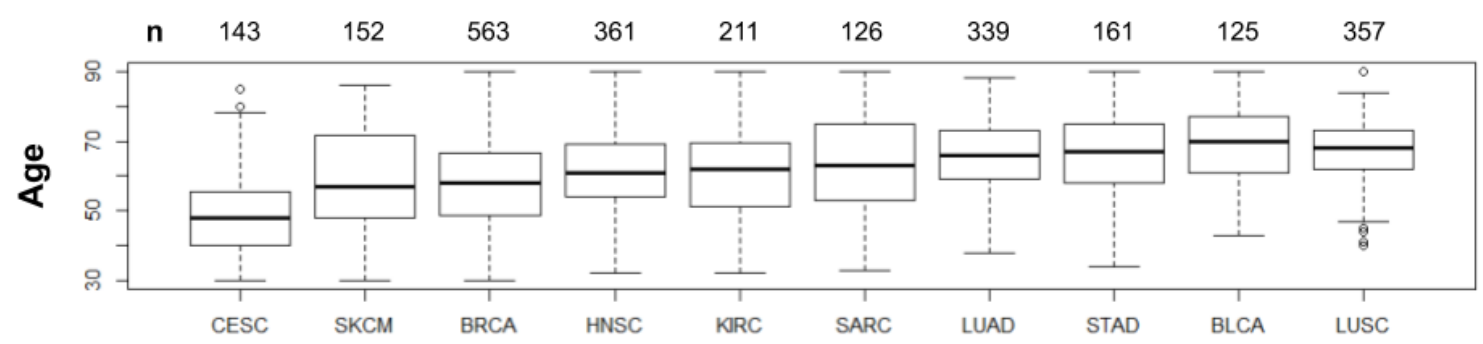

Cancer Type

Boxplot of the age demographic for each of the cancer types with at least 100 observations that were successfully deconvolved by immune cell type. The number of patients with each cancer is plotted above.

Supplemental Figure 4

A

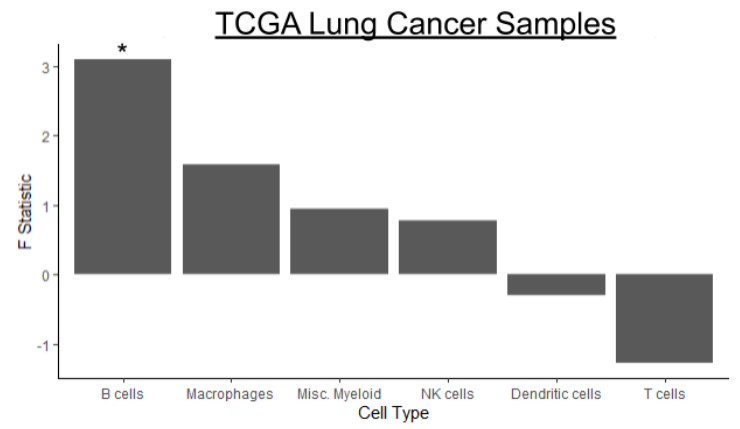

B

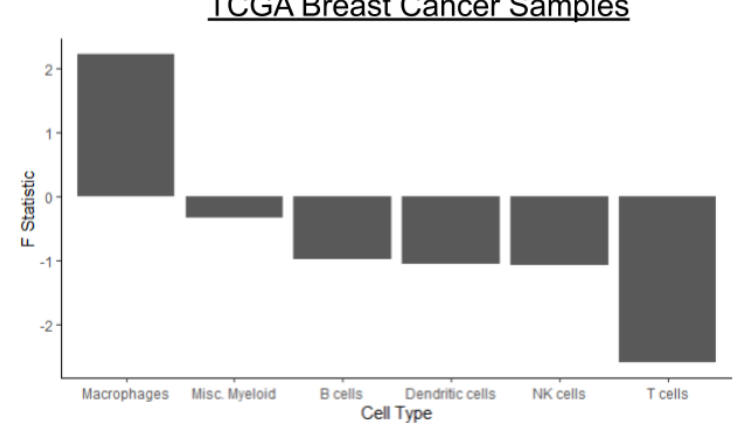

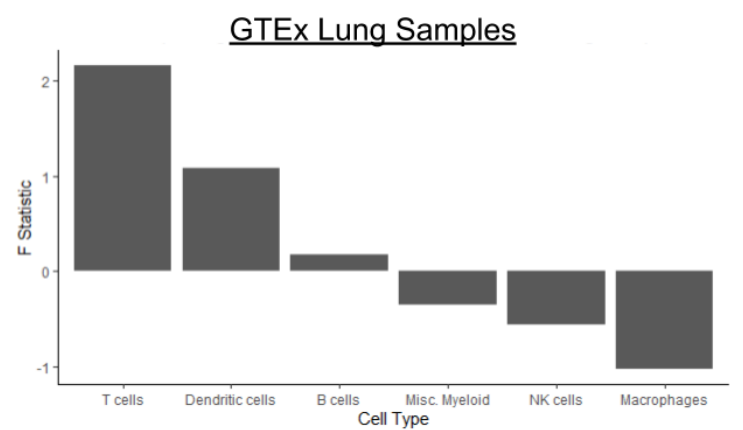

GTEx Breast Samples

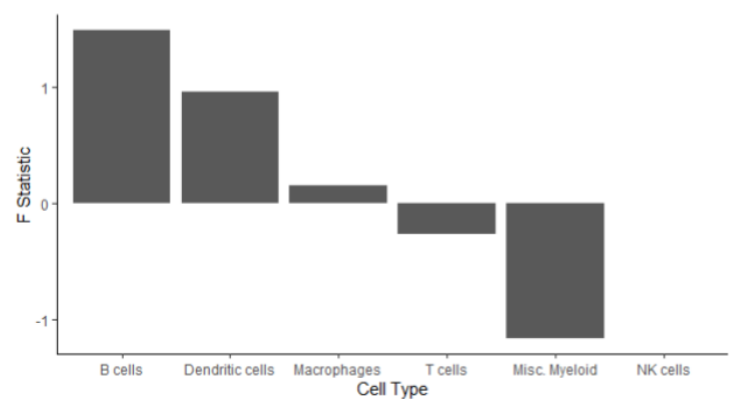

Association of immune cell type and age. The F statistic for the diagnosis age term of an ANOVA for each immune cell type (including age and sex as covariates) is used to compare $\mathbf{A}$ lung cancers to healthy lung tissue samples and $\mathbf{B}$ breast cancers to healthy breast tissue samples. * indicates a p-value less than 0.05 . 
Supplemental Figure 5

A

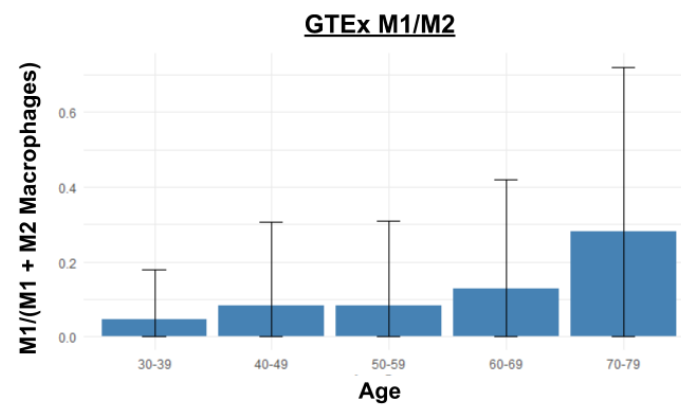

C

GTEx CD8T/CD4T

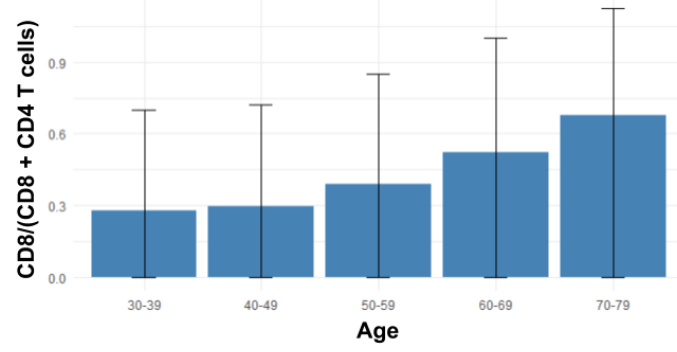

B

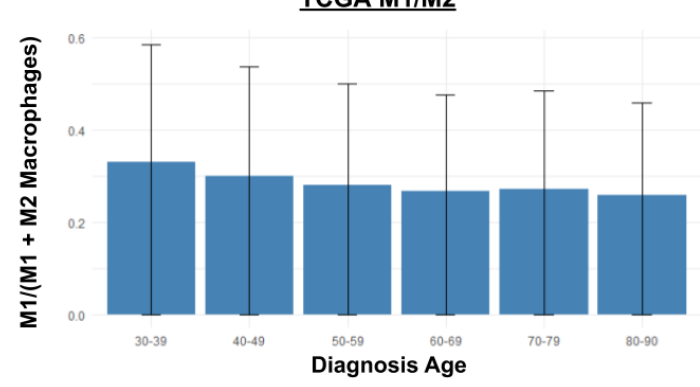

D

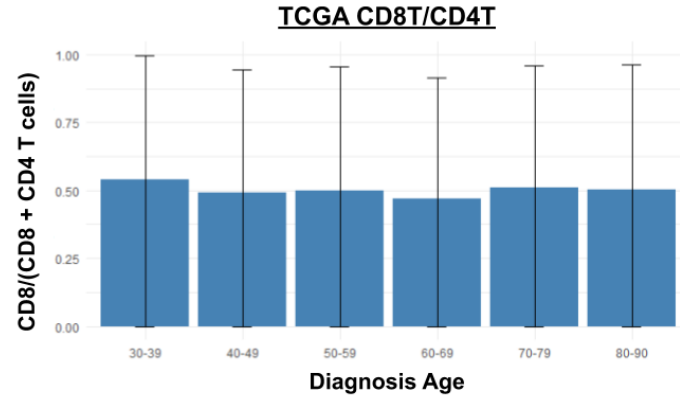

Comparing immune cell ratios of potential functional importance between TCGA and GTEx. A-B The proportion of M1 to M2 Macrophages in GTEx across tissues and TCGA pan-cancer, respectively. There is a significant increase in $M 1 / M 2$ ratio in GTEx tissues with age, while there is no significant shift in TCGA. C-D Proportion of CD8 T cells to CD4 T cells with age in GTEx across tissues and TCGA pan-cancer, respectively. There is a significant increase in CD8/CD4 ratio in GTEx tissues with age, while there is no significant shift in TCGA.

\section{Supplemental Figure 6}


A
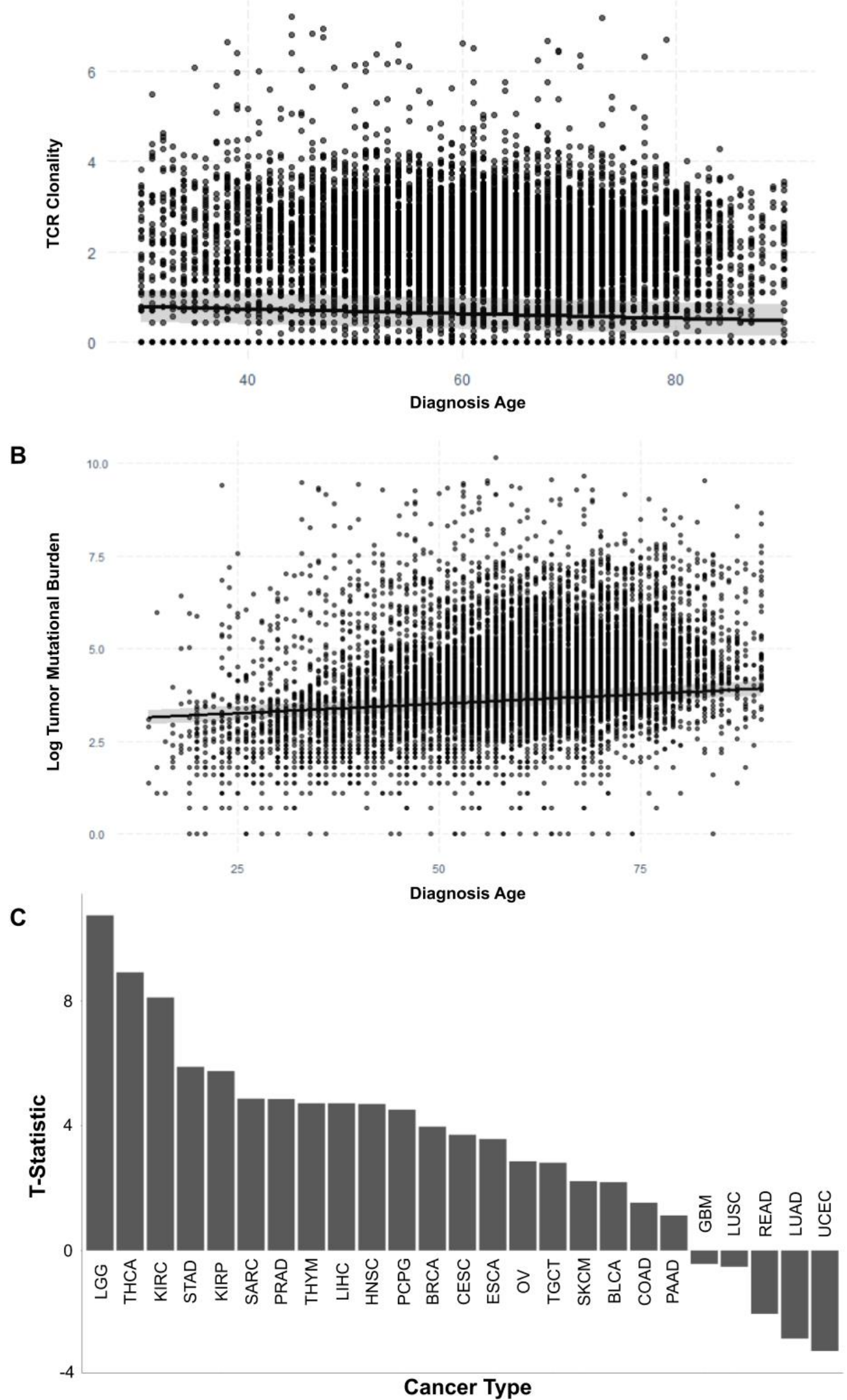
A Read normalized Shannon entropy of T cell receptor sequences plotted against age. The line fit to the data is a linear model of TCR clonality predicted by age, adjusted for sex and cancer type. B The log-normalized number of mutations found in each tumor sample is plotted against diagnosis age. The line fit to the data is a linear model of log-normalized number of mutations predicted by age, adjusted for sex and cancer type. C The T-statistic for the diagnosis age term of the linear model predicting the log normalized number of mutations for each cancer type.

\section{Supplemental Figure 7}

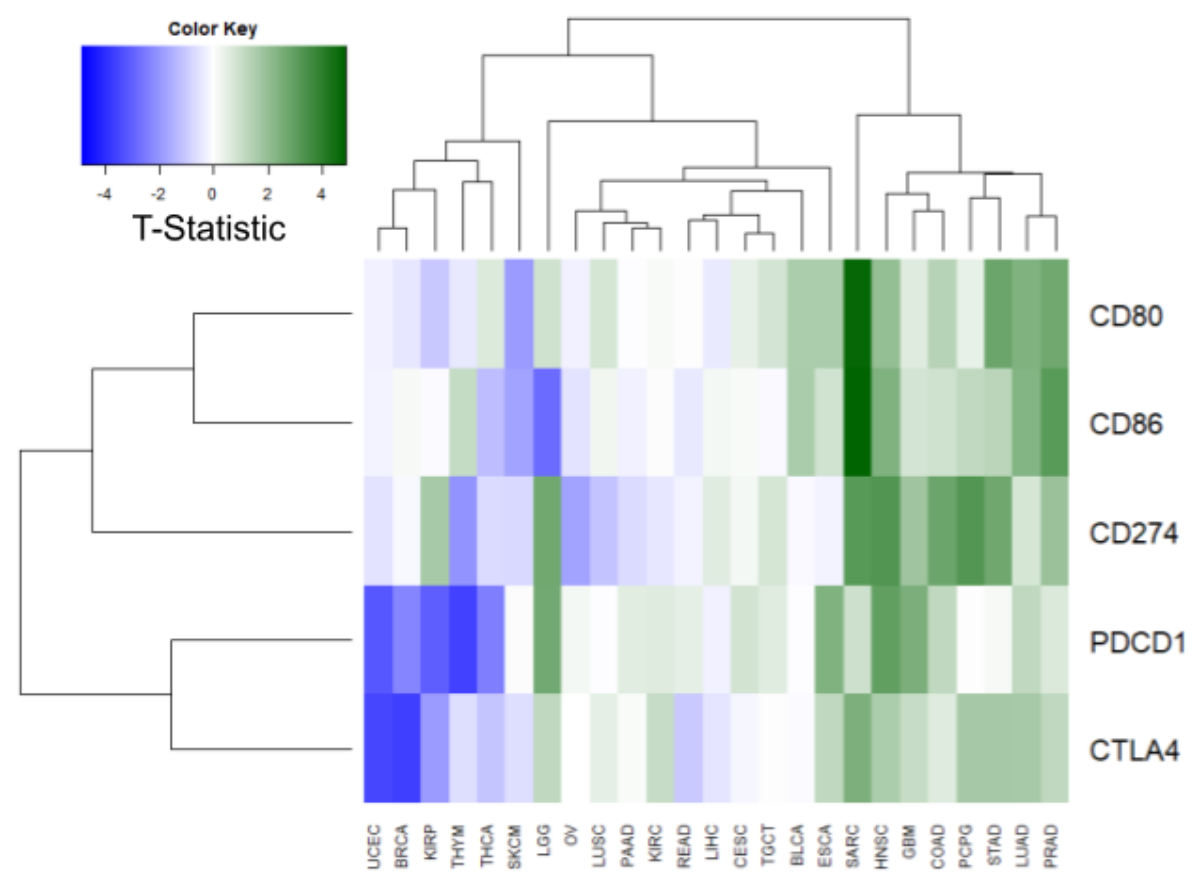

The association of immune checkpoint blockade genes with age across all cancer types with at least 100 samples in TCGA as determined by the t-statistic from limma-voom differential expression analysis. 
Supplemental Figure 8

A

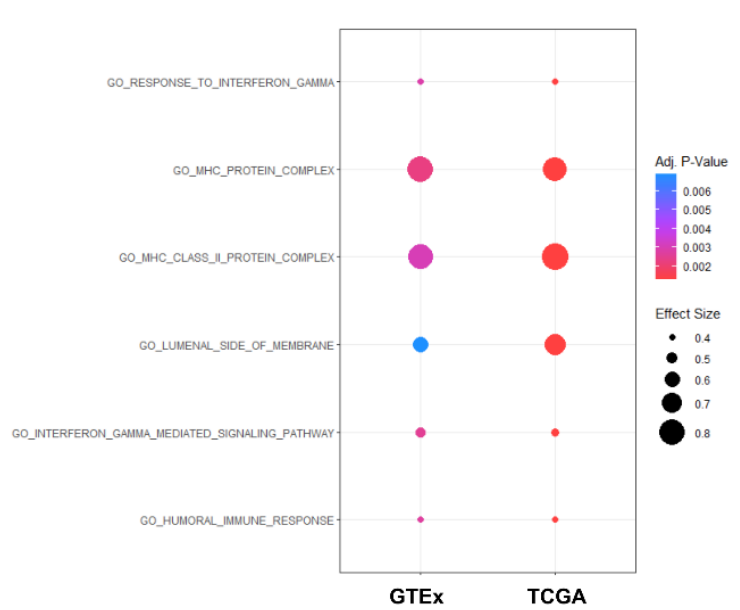

C

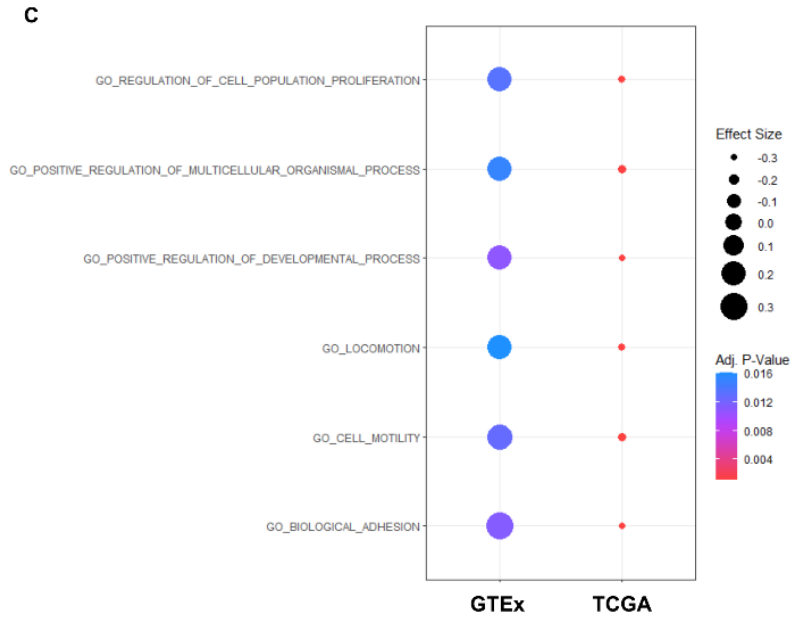

B

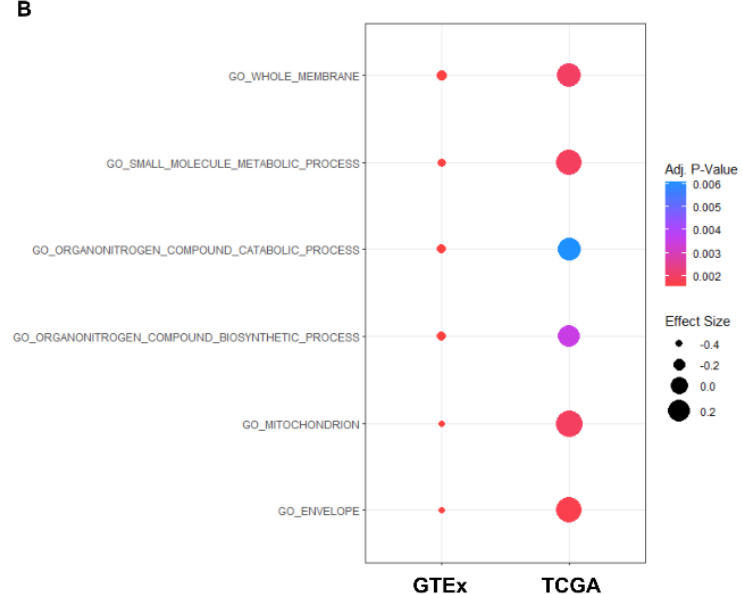

D

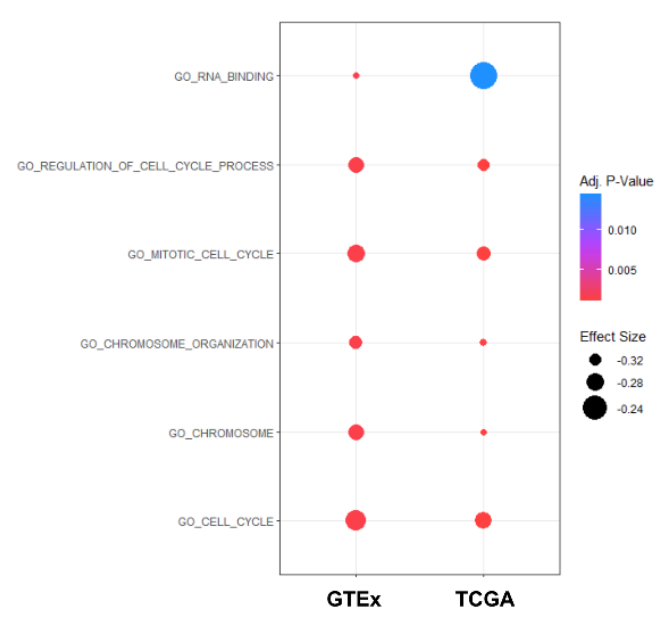

Dot plots representing genese set enrichment analysis for genes differentially expressed with age for both TCGA and GTEx. A Pathways significantly up in both TCGA and GTEx with age B Pathways significantly up in TCGA and down in GTEx with age $\mathbf{C}$ Pathways significantly up in GTEx and down in TCGA with age D Pathways significantly down in both TCGA and GTEx with age. 


\section{Supplemental Figure 9}

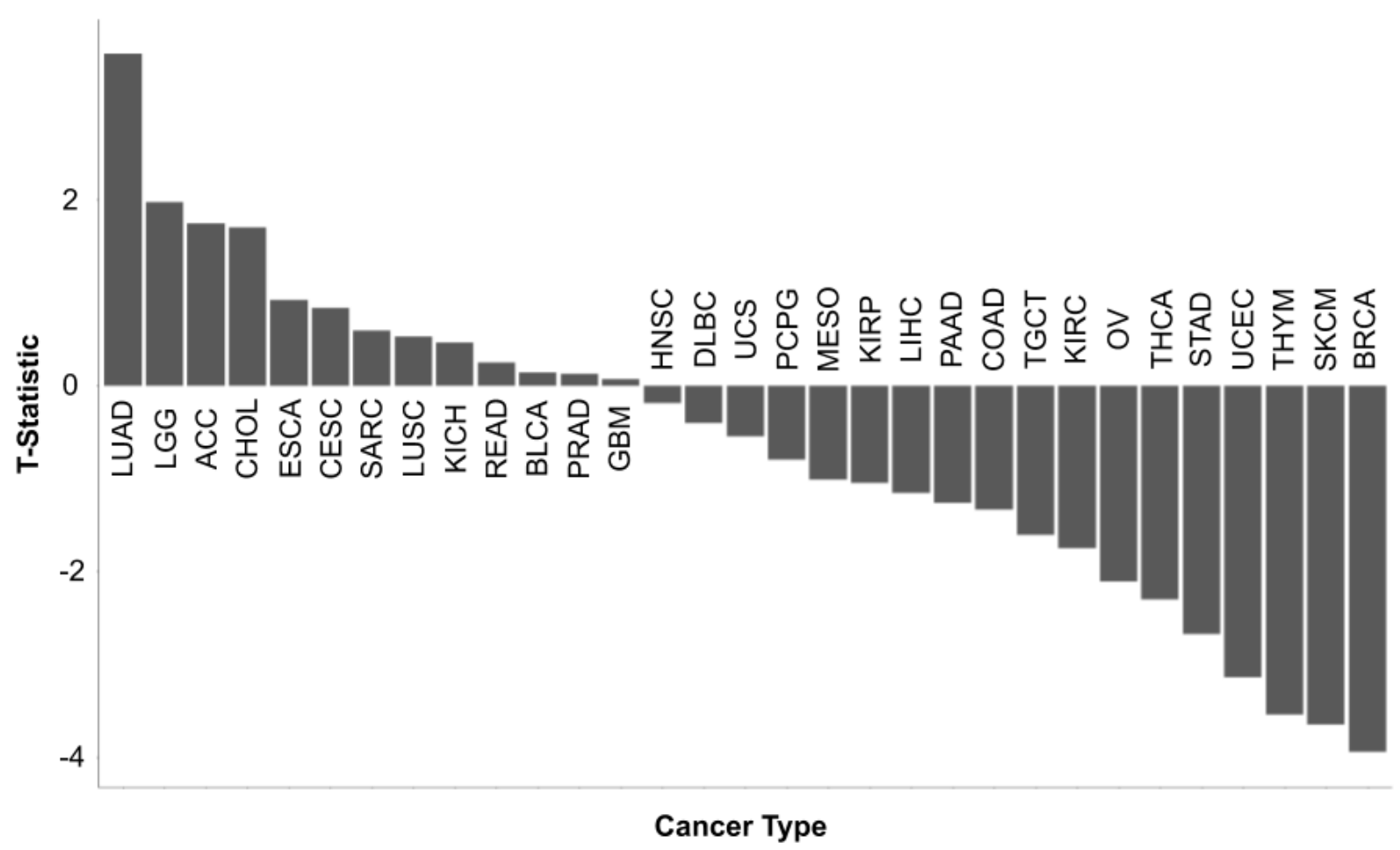

The association of TCR clonality and age is plotted for each cancer type in TCGA using the tstatistic from the diagnosis age term of a linear model fit to the TCR clonality of each cancer type, adjusting for sex as a covariate. 


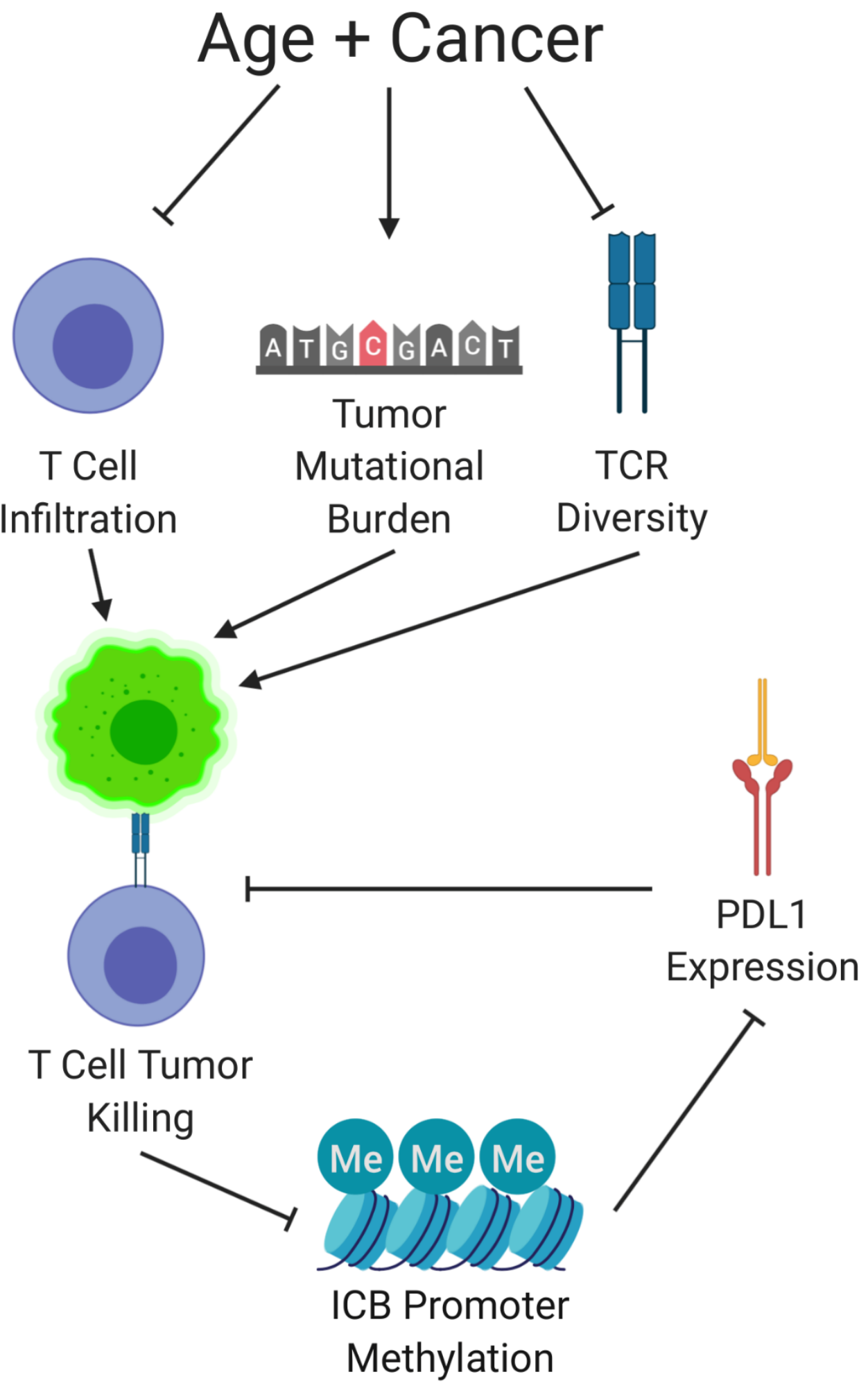

Hypothesized model for how the age-related effects we have observed affect T cell anti-tumor 
activity and expression of PDL1.

Supplemental Table 1

\begin{tabular}{|l|l|}
\hline \multicolumn{1}{|c|}{ Immune Cell Types } & \multicolumn{1}{c|}{ Immune Subtypes } \\
\hline T cells & $\begin{array}{l}\text { CD8 T cells, Naive CD4 T cells, Resting Memory Cd4 T cells, } \\
\text { Activated Memory CD4 T cells, Follicular Helper T cells, } \\
\text { Regulatory T cells, Gamma Delta T cells }\end{array}$ \\
\hline Macrophages & M0 Macrophages, M1 Macrophages, M2 Macrophages \\
\hline B cells & Naive B cells, Memory B cells, Plasma Cells \\
\hline NK cells & Resting NK cells, Activated NK cells \\
\hline Dendritic cells & Resting Dendritic cells, Activated Dendritic cells \\
\hline Misc. Myeloid cells & $\begin{array}{l}\text { Monocytes, Resting Mast cells, Activated Mast cells, Eosinophils, } \\
\text { Neutrophils }\end{array}$ \\
\hline
\end{tabular}

Immune cell types that were evaluated in relation to age for both TCGA tumor samples and GTEx normal samples.

Supplemental Table 2

\begin{tabular}{|l|l|l|l|}
\hline & \multicolumn{1}{|c|}{ F-statistic } & \multicolumn{1}{c|}{ p-value } & \multicolumn{1}{c|}{ q-value } \\
\hline T cells & 0.887307 & 0.470706 & 0.564847 \\
\hline Macrophages & 0.981287 & 0.416576 & 0.564847 \\
\hline B cells & 5.835976 & 0.000117 & 0.00035 \\
\hline NK cells & 19.34786 & $1.54 \mathrm{E}-15$ & $9.27 \mathrm{E}-15$ \\
\hline Dendritic cells & 2.265888 & 0.060047 & 0.120095 \\
\hline Misc. Myeloid & 0.647328 & 0.628807 & 0.628807 \\
\hline
\end{tabular}

F-statistic, $p$, and q-values for each immune cell term in an ANOVA for the effect of age on the abundance of each immune cell type. Tissue and sex were included in the model as covariates. 
Supplemental Table 3

\begin{tabular}{|c|l|l|l|}
\hline & & Up Expressed & Down Expressed \\
\hline $\begin{array}{c}\text { Age, Cancer Type } \\
\text { Adjusted }\end{array}$ & Up Methylated & 66 & $\mathbf{2 1 8}$ \\
\cline { 2 - 4 } & Down Methylated & $\mathbf{1 1 3}$ & 94 \\
\hline $\begin{array}{c}\text { Mutational Burden } \\
\text { Adjusted }\end{array}$ & Up Methylated & 62 & 135 \\
\cline { 2 - 5 } & Down Methylated & $\mathbf{1 2 9}$ & 86 \\
\hline
\end{tabular}

Number of genes differentially methylated and differentially expressed among immune related genes from TCGA samples. The genes with a canonical relationship between methylation and expression are in bold. The comparison is made both including and not including mutational burden as a covariate.

Supplemental Table 4

\begin{tabular}{|l|l|l|}
\hline & TCGA & GTEx \\
\hline Subjects & 8984 & 980 \\
\hline Males & 4367 & 653 \\
\hline Females & 4617 & 327 \\
\hline Age $20-29^{*}$ & N/A & 84 \\
\hline Age $30-39$ & 667 & 78 \\
\hline Age $40-49$ & 1226 & 153 \\
\hline Age $50-59$ & 2106 & 315 \\
\hline Age $60-69$ & 2453 & 317 \\
\hline Age $70-79$ & 1693 & 33 \\
\hline Age $80-90$ & 504 & 0 \\
\hline
\end{tabular}


Basic demographic information used as covariates in the analyses conducted in this study comparing the TCGA and GTEx cohorts.

*Age 20-29 was excluded from the TCGA patients collected (See Materials and Methods) and for comparison these individuals were excluded from the final analysis of GTEx data as well

Supplemental Table 5

\begin{tabular}{|l|l|}
\hline \multicolumn{1}{|c|}{ Tissue } & $\begin{array}{c}\text { Number } \\
\text { of } \\
\text { Samples }\end{array}$ \\
\hline $\begin{array}{l}\text { Adipose } \\
\text { Tissue }\end{array}$ & 1204 \\
\hline $\begin{array}{l}\text { Adrenal } \\
\text { Gland }\end{array}$ & 258 \\
\hline Bladder & 21 \\
\hline Blood & 929 \\
\hline Blood \\
Vessel & 1335 \\
\hline Brain & 2642 \\
\hline Breast & 459 \\
\hline Cervix \\
Uteri & 19 \\
\hline Colon & 779 \\
\hline Esophagus & 1445 \\
\hline \begin{tabular}{l} 
Sla \\
\hline
\end{tabular} & \\
\hline
\end{tabular}


bioRxiv preprint doi: https://doi.org/10.1101/2020.06.08.140764; this version posted June 8, 2020. The copyright holder for this preprint (which was not certified by peer review) is the author/funder, who has granted bioRxiv a license to display the preprint in perpetuity. It is made available under aCC-BY-NC 4.0 International license.

\begin{tabular}{|l|l|}
\hline $\begin{array}{l}\text { Fallopian } \\
\text { Tube }\end{array}$ & 9 \\
\hline Heart & 861 \\
\hline Kidney & 89 \\
\hline Liver & 226 \\
\hline Lung & 578 \\
\hline Muscle & 803 \\
\hline Nerve & 619 \\
\hline Ovary & 180 \\
\hline Pancreas & 328 \\
\hline Pituitary & 283 \\
\hline Prostate & 245 \\
\hline $\begin{array}{l}\text { Salivary } \\
\text { Gland }\end{array}$ & 162 \\
\hline Skin & 1809 \\
\hline $\begin{array}{l}\text { Small } \\
\text { Intestine }\end{array}$ & 187 \\
\hline Spleen & 241 \\
\hline Stomach & 359 \\
\hline
\end{tabular}




\begin{tabular}{|l|l|}
\hline Testis & 361 \\
\hline Thyroid & 653 \\
\hline Uterus & 142 \\
\hline Vagina & 156 \\
\hline
\end{tabular}

Number of RNA-seq samples from each tissue in GTEx data used.

Supplemental Table 6

\begin{tabular}{|l|l|}
\hline $\begin{array}{c}\text { TCGA } \\
\text { Cancer } \\
\text { Type } \\
\text { Acronym }\end{array}$ & $\begin{array}{c}\text { Number } \\
\text { of } \\
\text { Samples }\end{array}$ \\
\hline ACC & 64 \\
\hline BLCA & 405 \\
\hline BRCA & 1056 \\
\hline CESC & 270 \\
\hline CHOL & 35 \\
\hline COAD & 272 \\
\hline DLBC & 45 \\
\hline ESCA & 180 \\
\hline
\end{tabular}


bioRxiv preprint doi: https://doi.org/10.1101/2020.06.08.140764; this version posted June 8, 2020. The copyright holder for this preprint (which was not certified by peer review) is the author/funder, who has granted bioRxiv a license to display the preprint in perpetuity. It is made available under aCC-BY-NC 4.0 International license.

\begin{tabular}{|c|c|}
\hline HNSC & 507 \\
\hline $\mathrm{KICH}$ & 61 \\
\hline KIRC & 508 \\
\hline KIRP & 276 \\
\hline LAML & 158 \\
\hline LGG & 433 \\
\hline LIHC & 347 \\
\hline LUAD & 479 \\
\hline LUSC & 474 \\
\hline MESO & 86 \\
\hline OV & 292 \\
\hline PAAD & 177 \\
\hline PCPG & 157 \\
\hline PRAD & 480 \\
\hline READ & 88 \\
\hline SARC & 244 \\
\hline SKCM & 411 \\
\hline
\end{tabular}


bioRxiv preprint doi: https://doi.org/10.1101/2020.06.08.140764; this version posted June 8, 2020. The copyright holder for this preprint (which was not certified by peer review) is the author/funder, who has granted bioRxiv a license to display the preprint in perpetuity. It is made available under aCC-BY-NC 4.0 International license.

\begin{tabular}{|l|l|}
\hline STAD & 403 \\
\hline TGCT & 74 \\
\hline THCA & 433 \\
\hline THYM & 116 \\
\hline UCEC & 169 \\
\hline UCS & 57 \\
\hline UVM & 79 \\
\hline
\end{tabular}

Number of RNA-seq samples from each TCGA study in the TCGA data used in this work. 